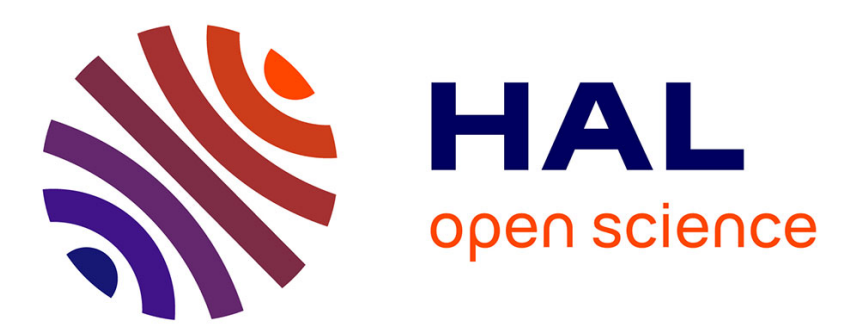

\title{
Boundary stabilization of elastodynamic systems. Part I: Rellich-type relations for a problem in elasticity involving singularities.
}

\author{
Romain Brossard, Jean-Pierre Lohéac
}

\section{To cite this version:}

Romain Brossard, Jean-Pierre Lohéac. Boundary stabilization of elastodynamic systems. Part I: Rellich-type relations for a problem in elasticity involving singularities.. 2007. hal-00196913v3

\section{HAL Id: hal-00196913 \\ https://hal.science/hal-00196913v3}

Preprint submitted on 12 Jun 2008

HAL is a multi-disciplinary open access archive for the deposit and dissemination of scientific research documents, whether they are published or not. The documents may come from teaching and research institutions in France or abroad, or from public or private research centers.
L'archive ouverte pluridisciplinaire HAL, est destinée au dépôt et à la diffusion de documents scientifiques de niveau recherche, publiés ou non, émanant des établissements d'enseignement et de recherche français ou étrangers, des laboratoires publics ou privés. 


\title{
Boundary stabilization of elastodynamic systems.
}

\section{Part I: Rellich-type relations for a problem in elasticity involving singularities.}

\author{
Brossard, R.*, Lohéac, J.-P. ${ }^{\dagger}$.
}

\begin{abstract}
For the Lamé's system, mixed boundary conditions generate singularities in the solution, mainly when the boundary of the domain is connected. We here prove Rellich relations involving these singularities.

These relations are useful in the problem of boundary stabilization of the elastodynamic system when using the multipliers method. This problem is studied in the second part [3].

Résumé. Pour le système de Lamé, des conditions au bord mixtes génèrent des singularités dans la solution, en particulier lorsque la frontière du domaine est connexe. Nous prouvons ici des relations de Rellich, en tenant compte de ces singularités.

Ces relations sont utiles pour obtenir la stabilisation frontière du système élastodynamique, en utilisant la méthode des multiplicateurs. Ce problème est étudié dans la deuxième partie [3].
\end{abstract}

\section{Introduction}

In this work, we present a detailed proof of a result which has been announced in [2] and some extension of this result.

Let $\Omega \subset \mathbb{R}^{n}$ be a bounded connected open set such that its boundary satisfies

$$
\partial \Omega=\partial \Omega_{D} \cup \partial \Omega_{N}, \quad \text { with }\left\{\begin{array}{l}
\partial \Omega_{D} \cap \partial \Omega_{N}=\emptyset \\
\operatorname{meas}\left(\partial \Omega_{D}\right) \neq 0 \\
\operatorname{meas}\left(\partial \Omega_{N}\right) \neq 0
\end{array}\right.
$$

We denote the boundary interface by $\Gamma=\overline{\partial \Omega_{D}} \cap \overline{\partial \Omega_{N}}$.

At a given $\mathbf{x} \in \partial \Omega$, we can consider $\boldsymbol{\nu}(\mathbf{x})$ the normal unit vector pointing outward of $\Omega$. For a regular vector field $\mathbf{v}$, we define the strain tensor and the stress tensor by

$$
\epsilon_{i j}(\mathbf{v})=\frac{1}{2}\left(\partial_{i} \mathbf{v}_{j}+\partial_{j} \mathbf{v}_{i}\right), \quad \sigma(\mathbf{v})=2 \mu \epsilon(\mathbf{v})+\lambda \operatorname{div}(\mathbf{v}) I_{n}
$$

where $\lambda$ and $\mu$ are the Lamé's coefficients and $I_{n}$ is the identity matrix of $\mathbb{R}^{n}$.

We introduce following Sobolev spaces: $\mathbb{L}^{2}(\Omega)=\left(\mathrm{L}^{2}(\Omega)\right)^{n}, \mathbb{H}^{s}(\Omega)=\left(\mathrm{H}^{s}(\Omega)\right)^{n}$, for every $s>0$, and $\mathbb{H}_{D}^{1}(\Omega)=\left\{\mathbf{v} \in \mathbb{H}^{1}(\Omega) / \mathbf{v}=\mathbf{0}\right.$, on $\left.\partial \Omega_{D}\right\}$.

We here consider the following mixed boundary problem:

$$
\begin{cases}-\operatorname{div}(\sigma(\mathbf{u}))=\mathbf{f}, & \text { in } \Omega \\ \mathbf{u}=\mathbf{g}, & \text { on } \partial \Omega_{D} \\ \sigma(\mathbf{u}) \boldsymbol{\nu}=\mathbf{h}, & \text { on } \partial \Omega_{N}\end{cases}
$$

*Université de Lyon, École Centrale de Lyon, Département Mathématiques Informatique, Institut Camille-Jordan, (C.N.R.S. U.M.R. 5208), 36 avenue Guy-de-Collongue, 69134 ÉCULLY Cedex, FRANCE, Romain.Brossard@ec-lyon.fr

†Université de Lyon, École Centrale de Lyon, Département Mathématiques Informatique, Institut Camille-Jordan, (C.N.R.S. U.M.R. 5208), 36 avenue Guy-de-Collongue, 69134 ÉCULLY Cedex, FRANCE, Jean-Pierre.Loheac@ec-lyon.fr 
where $\mathbf{f} \in \mathbb{L}^{2}(\Omega), \mathbf{g} \in \mathbb{H}^{3 / 2}\left(\partial \Omega_{D}\right)$ and $\mathbf{h} \in \mathbb{H}^{1 / 2}\left(\partial \Omega_{N}\right)$.

It is well-known that this problem admits a unique solution in $\mathbb{H}^{1}(\Omega)$.

Furthermore, singularities are generated in the solution when the boundary interface $\Gamma$ is non-empty. These singularities are described in [4, 9, 6] in the bi-dimensional case. Similar situations appear in Laplace problems and have been addressed by many authors (see for example [5, 1]).

We prove here integral relations for problem (2), of the type of those introduced in [11] by Rellich.

For a given point $\mathbf{x}_{0}$ in $\mathbb{R}^{n}$, we denote by $\mathbf{m}$ the following function: $\mathbf{m}(\mathbf{x})=\mathbf{x}-\mathbf{x}_{0}$.

For two vector fields, $\mathbf{v}_{1}$ and $\mathbf{v}_{2}$, let us define

$$
\Theta\left(\mathbf{v}_{1}, \mathbf{v}_{2}\right)=2\left(\sigma\left(\mathbf{v}_{1}\right) \boldsymbol{\nu}\right) \cdot\left((\mathbf{m} \cdot \nabla) \mathbf{v}_{2}\right)-(\mathbf{m} \cdot \boldsymbol{\nu})\left(\sigma\left(\mathbf{v}_{1}\right): \epsilon\left(\mathbf{v}_{2}\right)\right) .
$$

If the solution of problem (2) is regular enough (for example $\mathbb{H}^{2}$, then by means of two successive Green's formulæ, we obtain the following Rellich relation

$$
2 \int_{\Omega} \operatorname{div}(\sigma(\mathbf{u})) \cdot((\mathbf{m} \cdot \nabla) \mathbf{u}) d \mathbf{x}=(n-2) \int_{\Omega} \sigma(\mathbf{u}): \epsilon(\mathbf{u}) d \mathbf{x}+\int_{\partial \Omega} \Theta(\mathbf{u}, \mathbf{u}) d \boldsymbol{\gamma} .
$$

The aim of this paper is to generalize such a relation. It is organized as follows.

- In Section 1, we study the $n$-dimensional case when the interface is empty. In this case, the above Rellich relation holds (Theorem 1.1).

- In Section 2, we study the case of a bi-dimensional polygonal domain. Here, some additional terms can appear in the Rellich relation (Theorem 2.1).

- In Section 3, we generalize the previous result in the case of a smooth bi-dimensional domain (Theorem 3.1): in this case additional punctual terms appear.

- Finally, in Section 4, we study the case of a smooth $n$-dimensional domain. Here the additional term is an integral term along the interface $\Gamma$ (Theorem 4.1).

In three last sections, we need to analyze the local structure of the solution of mixed problem (2) in a neighborhood of the interface $\Gamma$. This is done thanks to results of previous works by P. Grisvard [4, 5, 6] and B. Mérouani 9 .

We emphasize that these Rellich relations are useful for proving boundary stabilization results for the elastodynamic system by using the multiplier method as well as in [5, 7, 1]. This work is developed in the second part.

\section{The case of a $n$-dimensional domain without interface}

In this section, we assume (1) and that $\partial \Omega_{N}$ and $\partial \Omega_{D}$ are defined by means of above function $\mathbf{m}$,

$$
\partial \Omega_{N}=\{\mathbf{x} \in \partial \Omega / \mathbf{m}(\mathbf{x}) \cdot \boldsymbol{\nu}(\mathbf{x})>0\}, \quad \partial \Omega_{D}=\{\mathbf{x} \in \partial \Omega / \mathbf{m}(\mathbf{x}) \cdot \boldsymbol{\nu}(\mathbf{x})<0\},
$$

so that we get (see Figure 1)

$$
\Gamma=\emptyset
$$

We obtain the following Rellich-type relation.

Theorem 1.1 - Let $\Omega \subset \mathbb{R}^{n}$ be a bounded connected open set, where $n \geq 2$, such that its boundary is of class $\mathcal{C}^{2}$ and satisfies (1) and (5). Let $\mathbf{u} \in \mathbb{H}^{1}(\Omega)$ be the solution of problem (2) with

$$
\mathbf{f} \in \mathbb{L}^{2}(\Omega), \quad \mathbf{g} \in \mathbb{H}^{3 / 2}\left(\partial \Omega_{D}\right), \quad \mathbf{h} \in \mathbb{H}^{1 / 2}\left(\partial \Omega_{N}\right) .
$$

Then $\Theta(\mathbf{u}, \mathbf{u})$ (see (3)) belongs to $\mathrm{L}^{1}(\partial \Omega)$ and

$$
2 \int_{\Omega} \operatorname{div}(\sigma(\mathbf{u})) \cdot((\mathbf{m} \cdot \nabla) \mathbf{u}) d \mathbf{x}=(n-2) \int_{\Omega} \sigma(\mathbf{u}): \epsilon(\mathbf{u}) d \mathbf{x}+\int_{\partial \Omega} \Theta(\mathbf{u}, \mathbf{u}) d \boldsymbol{\gamma} .
$$




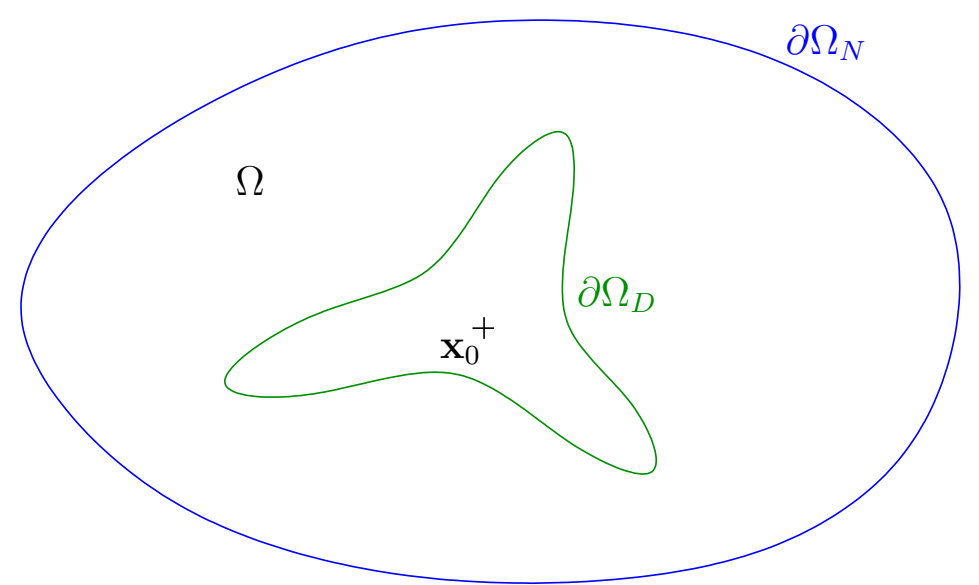

Figure 1: an example of a domain $\Omega$ without interface.

Proof. Our proof is composed of two steps: first we prove a hidden regularity result, secondly we apply Green's formula.

- First step. Let us prove that $\mathbf{u} \in \mathbb{H}^{2}(\Omega)$.

- Interior regularity. For every open subset $\mathcal{V}$ such that $\overline{\mathcal{V}} \subset \Omega$, multiplying by a cut-off function leads us to the following problem

$$
-\operatorname{div}(\tilde{\sigma}(\tilde{\mathbf{u}}))=\mathbf{f}, \quad \text { in } \mathbb{R}^{n} .
$$

We know that for this problem, $\mathbf{f} \in \mathbb{L}^{2}\left(\mathbb{R}^{n}\right)$ implies: $\tilde{\mathbf{u}} \in \mathbb{H}^{2}\left(\mathbb{R}^{n}\right)$. Hence we get: $\mathbf{u} \in \mathbb{H}^{2}(\mathcal{V})$.

- Boundary regularity. Let $\mathbf{x}$ be a point of $\partial \Omega_{D}$. We introduce a neighborhood $\mathcal{V}$ of $\mathbf{x}$ in $\mathbb{R}^{n}$ such that $\mathcal{V} \cap \partial \Omega=\mathcal{V} \cap \partial \Omega_{D}$. Multiplying by a cut-off function and changing the coordinates leads us to some problem in the following form

$$
\begin{cases}-\operatorname{div}(\tilde{\sigma}(\tilde{\mathbf{u}}))=\overline{\mathbf{f}}, & \text { in } \mathbb{R}_{+}^{n}, \\ \tilde{\mathbf{u}}=\overline{\mathbf{g}}, & \text { on } \mathbb{R}^{n-1} \times\{0\},\end{cases}
$$

where $\mathbb{R}_{+}^{n}=\left\{\left(x_{1}, \ldots, x_{n}\right) \in \mathbb{R}^{n} / x_{n}>0\right\}, \overline{\mathbf{f}} \in \mathbb{L}^{2}\left(\mathbb{R}_{+}^{n}\right)$ and $\overline{\mathbf{g}} \in \mathbb{H}^{3 / 2}\left(\mathbb{R}^{n-1}\right)$.

Using a trace result, we can build $\tilde{\mathbf{U}} \in \mathbb{H}^{2}\left(\mathbb{R}_{+}^{n}\right)$ such that $\tilde{\mathbf{U}}=\overline{\mathbf{g}}$, on $\mathbb{R}^{n-1} \times\{0\}$.

We write $\tilde{\mathbf{f}}=\operatorname{div}(\tilde{\sigma}(\tilde{\mathbf{U}}))-\operatorname{div}(\tilde{\sigma}(\tilde{\mathbf{u}})) . \tilde{\mathbf{f}} \in \mathbb{L}^{2}\left(\mathbb{R}_{+}^{n}\right)$ and $\mathbf{U}=\tilde{\mathbf{u}}-\tilde{\mathbf{U}}$ satisfies

$$
\begin{cases}-\operatorname{div}(\tilde{\sigma}(\mathbf{U}))=\tilde{\mathbf{f}}, & \text { in } \mathbb{R}_{+}^{n} \\ \mathbf{U}=\mathbf{0}, & \text { on } \mathbb{R}^{n-1} \times\{0\} .\end{cases}
$$

Using the differential quotients method, we prove that $\tilde{\mathbf{u}} \in \mathbb{H}^{2}\left(\mathbb{R}_{+}^{n}\right)$. Hence we get: $\mathbf{u} \in \mathbb{H}^{2}(\Omega \cap \mathcal{V})$.

For a point $\mathbf{x}$ of $\partial \Omega_{N}$, a similar method leads to: $\mathbf{u}$ is $\mathbb{H}^{2}$ in some neighborhood of $\mathbf{x}$.

Finally, since $\bar{\Omega}$ is compact, we can conclude that $\mathbf{u}$ belongs to $\mathbb{H}^{2}(\Omega)$.

- Second step. Green's formulæ.

We may now apply Green's formula a first time,

$2 \int_{\Omega} \operatorname{div}(\sigma(\mathbf{u})) \cdot((\mathbf{m} \cdot \nabla) \mathbf{u}) d \mathbf{x}=2 \int_{\partial \Omega}(\sigma(\mathbf{u}) \boldsymbol{\nu}) \cdot((\mathbf{m} \cdot \nabla) \mathbf{u}) d \boldsymbol{\gamma}-2 \int_{\Omega} \sigma(\mathbf{u}): \nabla((\mathbf{m} \cdot \nabla) \mathbf{u}) d \mathbf{x}$

$$
=2 \int_{\partial \Omega}(\sigma(\mathbf{u}) \boldsymbol{\nu}) \cdot((\mathbf{m} \cdot \nabla) \mathbf{u}) d \boldsymbol{\gamma}-2 \int_{\Omega} \sigma(\mathbf{u}): \epsilon(\mathbf{u}) d \mathbf{x}-\int_{\Omega} \nabla(\sigma(\mathbf{u}): \epsilon(\mathbf{u})) \cdot \mathbf{m} d \mathbf{x},
$$

and a second time,

$$
\begin{aligned}
2 \int_{\Omega} \operatorname{div}(\sigma(\mathbf{u})) \cdot((\mathbf{m} \cdot \nabla) \mathbf{u}) d \mathbf{x} & =2 \int_{\partial \Omega}(\sigma(\mathbf{u}) \boldsymbol{\nu}) \cdot((\mathbf{m} \cdot \nabla) \mathbf{u}) d \boldsymbol{\gamma}-2 \int_{\Omega} \sigma(\mathbf{u}): \epsilon(\mathbf{u}) d \mathbf{x} \\
& -\int_{\partial \Omega}(\mathbf{m} \cdot \boldsymbol{\nu})(\sigma(\mathbf{u}): \epsilon(\mathbf{u})) d \boldsymbol{\gamma}+n \int_{\Omega} \sigma(\mathbf{u}): \epsilon(\mathbf{u}) d \mathbf{x} .
\end{aligned}
$$

Hence, we get the required results. 


\section{The case of a bi-dimensional polygonal domain}

We here study the case of a convex polygonal domain $\Omega \subset \mathbb{R}^{2}$. We assume that its boundary $\partial \Omega$ satisfies (1) and furthermore, the interface $\Gamma$ is composed of two points,

$$
\Gamma=\left\{\mathbf{s}_{1}, \mathbf{s}_{2}\right\}
$$

These points $\mathbf{s}_{1}$ and $\mathbf{s}_{2}$ will be considered as two vertices of $\partial \Omega$, (see Figure 2).

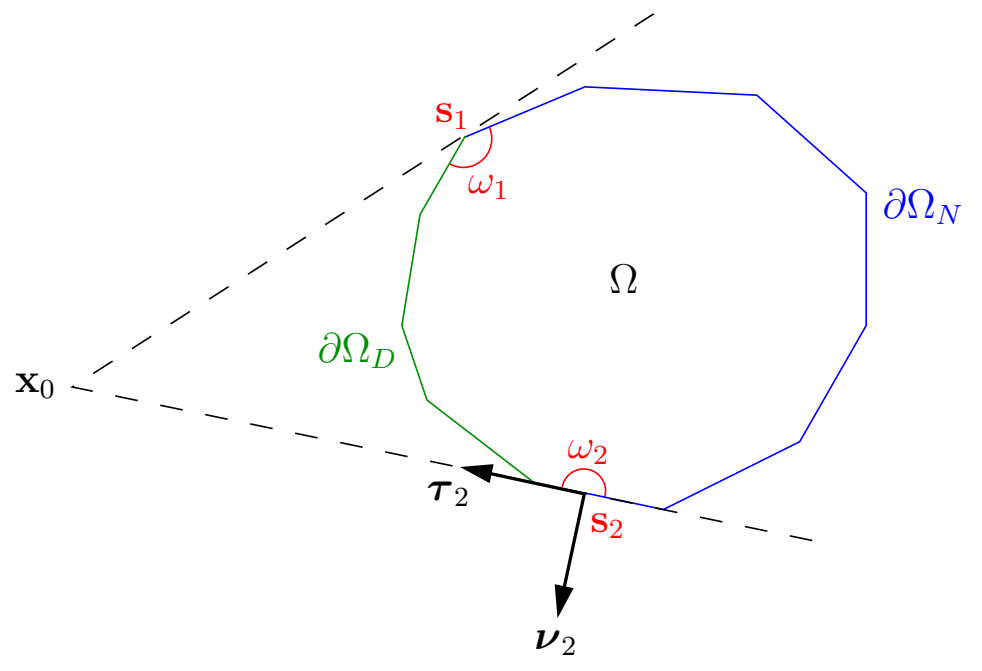

Figure 2: a polygonal domain $\Omega$ with a non-empty interface $\Gamma=\left\{\mathbf{s}_{1}, \mathbf{s}_{2}\right\}$.

We assume moreover that there exists $\mathbf{x}_{0} \in \mathbb{R}^{2}$ such that

$$
\mathbf{m} \cdot \boldsymbol{\nu} \geq 0, \quad \text { on } \partial \Omega_{N}, \quad \text { and } \quad \mathbf{m} \cdot \boldsymbol{\nu} \leq 0, \quad \text { on } \partial \Omega_{D} .
$$

Let us recall that changing boundary conditions generates singularities in the solution of our problem. We will denote by $c(\mathbf{s})$ the coefficient of the singularity associated to point $\mathbf{s}$.

At each point $\mathbf{s}_{i}$, we define $\omega\left(\mathbf{s}_{i}\right)$ the angle of $\Omega$. If $\omega\left(\mathbf{s}_{i}\right)=\pi$, we denote by $\boldsymbol{\tau}\left(\mathbf{s}_{i}\right)$ the unit tangent vector to $\partial \Omega$ pointing toward $\partial \Omega_{D}$, (see Figure 2). Let us observe that condition $(7)$ leads to

$$
\omega\left(\mathbf{s}_{i}\right)=\pi \Longrightarrow \mathbf{m}\left(\mathbf{s}_{i}\right) \cdot \boldsymbol{\nu}\left(\mathbf{s}_{i}\right)=\mathbf{0} .
$$

Let us define

$$
\Upsilon=8 \frac{(2 \mu+\lambda)(3 \mu+\lambda)}{\pi \mu}\left(\pi^{2}+\ln ^{2}\left(\frac{3 \mu+\lambda}{\mu+\lambda}\right)\right) .
$$

We here obtain a Rellich-type relation in the following form:

Theorem 2.1 - Let $\Omega \subset \mathbb{R}^{2}$ be a bounded convex polygonal open set such that its boundary satisfies (1), (6) and (8). Let $\mathbf{u} \in \mathbb{H}^{1}(\Omega)$ be the solution of problem (2) with

$$
\mathbf{f} \in \mathbb{L}^{2}(\Omega), \quad \mathbf{g} \in \mathbb{H}^{3 / 2}\left(\partial \Omega_{D}\right), \quad \mathbf{h} \in \mathbb{H}^{1 / 2}\left(\partial \Omega_{N}\right) .
$$

Then $\Theta(\mathbf{u}, \mathbf{u})$ belongs to $\mathrm{L}^{1}(\partial \Omega)$ and

$$
2 \int_{\Omega} \operatorname{div}(\sigma(\mathbf{u})) \cdot((\mathbf{m} \cdot \nabla) \mathbf{u}) d \mathbf{x}=\int_{\partial \Omega} \Theta(\mathbf{u}, \mathbf{u}) d \boldsymbol{\gamma}+\Upsilon \sum_{\substack{\mathbf{s} \in\left\{\mathbf{s}_{1}, \mathbf{s}_{2}\right\} \\ \omega(\mathbf{s})=\pi}} c(\mathbf{s})^{2}(\mathbf{m}(\mathbf{s}) \cdot \boldsymbol{\tau}(\mathbf{s})),
$$

where $c(\mathbf{s})$ is the singularity coefficient of $\mathbf{u}$ at point $\mathbf{s}$. 
We emphasize that in above Rellich relation, singular term $c(\mathbf{s})^{2}(\mathbf{m}(\mathbf{s}) \cdot \boldsymbol{\tau}(\mathbf{s}))$ appears only if $\omega(\mathbf{s})=\pi$. In order to prove this Theorem, we use results of P. Grisvard and B. Merouani [4, 9, 6, and we proceed as well as in [5].

Proof. As well as for Theorem 1.1, we can prove that for every open set $\mathcal{V}$ such that $\overline{\mathcal{V}} \subset \Omega, \mathbf{u} \in \mathbb{H}^{2}(\mathcal{V})$. Similarly, for every $\mathbf{x} \in \partial \Omega \backslash \Gamma$ such that $\mathbf{x}$ is not a vertex, there exists a neighborhood $\mathcal{V}$ of $\mathbf{x}$ such that $\mathbf{u} \in \mathbb{H}^{2}(\mathcal{V} \cap \Omega)$.

For a vertex $\tilde{\mathbf{s}} \in \partial \Omega \backslash \Gamma$, we use [4, 9, 6] to prove that there exists a neighborhood $\mathcal{V}$ of $\tilde{\mathbf{s}}$ such that $\mathbf{u} \in \mathbb{H}^{2}(\mathcal{V} \cap \Omega)$.

Now consider $\mathbf{s} \in \Gamma$. Let $\gamma_{0}$ and $\gamma_{1}$ be the adjacent edges to $\mathbf{s}$. Using a cut-off function, we can suppose that $\mathbf{u}$ vanishes outside a disk $D(\mathbf{s}, \delta)$. We can suppose that $\delta$ is small enough, so that $D(\mathbf{s}, \delta) \cap \partial \Omega \subset \gamma_{0} \cup \gamma_{1}$. We take some $\varepsilon>0$ and define (see Figure 3 )

$$
\begin{array}{ll}
\mathcal{H}_{\varepsilon}=\{(r, \theta) \in(\varepsilon,+\infty) \times(0, \omega)\}, & \tilde{\Gamma}_{\varepsilon}=\{(\varepsilon, \theta) / \theta \in(0, \omega)\}, \\
\partial \mathcal{H}_{D \varepsilon}=\{(r, 0) / r>\varepsilon\}, & \partial \mathcal{H}_{N \varepsilon}=\{(r, \omega) / r>\varepsilon\},
\end{array}
$$

so that $\partial \mathcal{H}_{\varepsilon}=\partial \mathcal{H}_{D \varepsilon} \cup \partial \mathcal{H}_{N \varepsilon} \cup \tilde{\Gamma}_{\varepsilon}$.

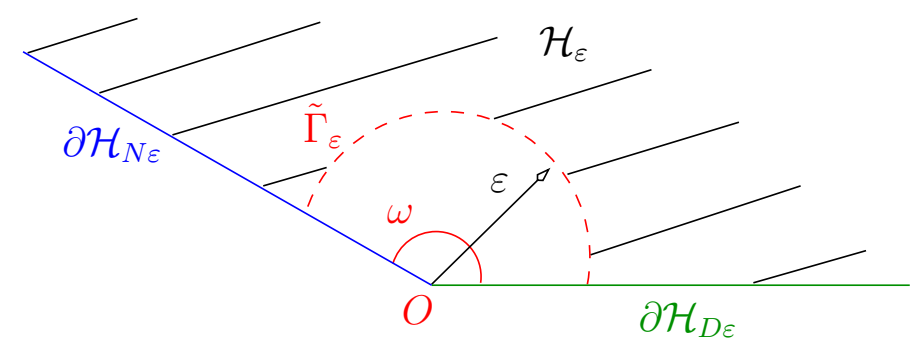

Figure 3: an example of set $\mathcal{H}_{\varepsilon}$ for $\omega<\pi$.

Then $\mathbf{u} \in \mathbb{H}^{2}\left(\mathcal{H}_{\varepsilon}\right)$ and Theorem 1.1 gives

$$
2 \int_{\mathcal{H}_{\varepsilon}} \operatorname{div}(\sigma(\mathbf{u})) \cdot((\mathbf{m} \cdot \nabla) \mathbf{u}) d \mathbf{x}=\int_{\partial \mathcal{H}_{\varepsilon}} \Theta(\mathbf{u}, \mathbf{u}) d \boldsymbol{\gamma} .
$$

Using Lebesgue's Theorem, we easily get

$$
\int_{\mathcal{H}_{\varepsilon}} \operatorname{div}(\sigma(\mathbf{u})) \cdot((\mathbf{m} \cdot \nabla) \mathbf{u}) d \mathbf{x} \underset{\varepsilon \rightarrow 0}{\longrightarrow} \int_{\mathcal{H}} \operatorname{div}(\sigma(\mathbf{u})) \cdot((\mathbf{m} \cdot \nabla) \mathbf{u}) d \mathbf{x} .
$$

We now consider the convergence of the right-hand side of $(9)$. Projecting method and changing coordinates leads us to consider the following problem

$$
\begin{cases}-\operatorname{div}(\sigma(\tilde{\mathbf{u}}))=\tilde{\mathbf{f}}, & \text { in } \mathcal{H} \\ \tilde{\mathbf{u}}=\mathbf{0}, & \text { on } \partial \mathcal{H}_{D} \\ \sigma(\tilde{\mathbf{u}}) \boldsymbol{\nu}=\mathbf{0}, & \text { on } \partial \mathcal{H}_{N}\end{cases}
$$

where $\mathcal{H}=\left\{(r, \theta) \in \mathbb{R}_{+}^{*} \times(0, \omega)\right\}, \partial \mathcal{H}_{D}=\{(r, 0) / r>0\}, \partial \mathcal{H}_{N}=\{(r, \omega) / r>0\}$, in polar coordinates, and where $\tilde{\mathbf{f}} \in \mathbb{L}^{2}(\mathcal{H})$.

Hence, we can write $\mathbf{u}=\mathbf{U}+\tilde{\mathbf{u}}$ where $\mathbf{U} \in \mathbb{H}^{2}(\mathcal{H})$. We use the structure of the solution of $(10)$ given in [4, 9, 6] and we now consider two cases.

\section{$2.1 \quad$ First case: $\omega<\pi$}

Let us denote by $\nu$ the Poisson's coefficient of the system, i.e. $\nu=\frac{1}{2} \frac{\lambda}{\lambda+\mu}$.

Following [4, 9, 6, we have to consider this equation in $\alpha$,

$$
\sin ^{2}(\alpha \omega)=\frac{4(1-\nu)^{2}-\alpha^{2} \sin ^{2} \omega}{3-4 \nu} .
$$


Let $\left(\alpha_{i}\right)_{i=1, \ldots, K}$ be the sequence of complex roots of $(11$ such that $\Re \alpha \in(0,1]$. From [4, 9, 6], we get

$$
\exists \tilde{\mathbf{u}}_{R} \in \mathbb{H}^{2}(\mathcal{H}) / \quad \forall i \in[1, K], \exists\left(\mathbf{v}_{i}^{1}, \mathbf{v}_{i}^{2}\right) \in\left(C^{\infty}([0, \omega], \mathbb{C})\right)^{2}: \quad \tilde{\mathbf{u}}=\tilde{\mathbf{u}}_{R}+\sum_{i=1}^{K} \Re\left[r^{\alpha_{i}}\left(\mathbf{v}_{i}^{1}(\theta)+\ln (r) \mathbf{v}_{i}^{2}(\theta)\right)\right]
$$

Writing $\mathbf{u}_{R}=\mathbf{U}+\tilde{\mathbf{u}}_{R}$, we get

$$
\mathbf{u}=\mathbf{u}_{R}+\sum_{i=1}^{K} \Re\left[r^{\alpha_{i}}\left(\mathbf{v}_{i}^{1}(\theta)+\ln (r) \mathbf{v}_{i}^{2}(\theta)\right)\right]
$$

The main point of our proof lies in the following Lemma which will be proved at the end of this section.

Lemma $2.1-\forall i \in[1, K], \Re \alpha_{i}>\frac{1}{2}$.

Hence, for the Rellich-type relation, there is no corrective term. We then prove

$$
\int_{\partial \mathcal{H}_{\varepsilon}} \Theta(\mathbf{u}, \mathbf{u}) d \gamma \underset{\varepsilon \rightarrow 0}{\longrightarrow} \int_{\partial \mathcal{H}} \Theta(\mathbf{u}, \mathbf{u}) d \gamma
$$

We know that $\mathbf{u}$ can be written in the form 12 . Using this form, we expand the left-hand side in (13) and we have to consider $\mathbf{u}_{1}$ and $\mathbf{u}_{2}$ two functions taken among the following ones: $\mathbf{u}_{R}, \Re\left[r^{\alpha_{i}} \mathbf{v}_{i}^{1}(\theta)\right]$, $\Re\left[r^{\alpha_{i}} \ln (r) \mathbf{v}_{i}^{2}(\theta)\right]$.

We have: $\exists C>0:\left|\Theta\left(\mathbf{u}_{1}, \mathbf{u}_{2}\right)\right| \leq C\left|\nabla \mathbf{u}_{1}\right|\left|\nabla \mathbf{u}_{2}\right|$.

- First case: $\mathbf{u}_{1}=\mathbf{u}_{2}=\mathbf{u}_{R}$

In this case, we have: $\mathbf{u}_{R} \in \mathbb{H}^{2}(\mathcal{H})$ and we may use Lebesgue's Theorem to get the result.

- Second case: $\mathbf{u}_{1}=\mathbf{u}_{R}$ and $\mathbf{u}_{2}=\Re\left[r^{\alpha_{i}} \mathbf{v}_{i}^{1}(\theta)\right]$

We have: $\exists C>0$ : $\left|\nabla \mathbf{u}_{2}\right| \leq C r^{\Re \alpha_{i}-1}$.

Hence, $\exists C>0:\left|\Theta\left(\mathbf{u}_{1}, \mathbf{u}_{2}\right)\right| \leq C\left|\nabla \mathbf{u}_{1}\right| r^{\Re \alpha_{i}-1}$.

Now, with lemma 2.1, $\Re \alpha_{i}-1>-1 / 2$. Then we may apply Lebesgue's Theorem and get

$$
\int_{\partial \mathcal{H}_{D \varepsilon}} \Theta\left(\mathbf{u}_{1}, \mathbf{u}_{2}\right) d \boldsymbol{\gamma} \underset{\varepsilon \rightarrow 0}{\longrightarrow} \int_{\partial \mathcal{H}_{D}} \Theta\left(\mathbf{u}_{1}, \mathbf{u}_{2}\right) d \boldsymbol{\gamma}, \quad \int_{\partial \mathcal{H}_{N \varepsilon}} \Theta\left(\mathbf{u}_{1}, \mathbf{u}_{2}\right) d \boldsymbol{\gamma} \underset{\varepsilon \rightarrow 0}{\longrightarrow} \int_{\partial \mathcal{H}_{N}} \Theta\left(\mathbf{u}_{1}, \mathbf{u}_{2}\right) d \boldsymbol{\gamma}
$$

Moreover, there exists $C>0$ such that

$$
\left|\int_{\tilde{\Gamma}_{\varepsilon}} \Theta\left(\mathbf{u}_{1}, \mathbf{u}_{2}\right) d \gamma\right|=\varepsilon\left|\int_{0}^{\omega} \Theta\left(\mathbf{u}_{1}, \mathbf{u}_{2}\right) d \theta\right| \leq C \varepsilon^{\Re \alpha_{i}} \underset{\varepsilon \rightarrow 0}{\longrightarrow} 0
$$

since $\Re \alpha_{i}>1 / 2$. Then 13 is satisfied.

Remark. We get a similar result with $\mathbf{u}_{2}=\Re\left[r^{\alpha_{i}} \ln (r) \mathbf{v}_{i}^{2}(\theta)\right]$. We also get the same result when reversing the roles of $\mathbf{u}_{1}$ and $\mathbf{u}_{2}$.

- Third case : $\mathbf{u}_{1}=\Re\left[r^{\alpha_{i}} \mathbf{v}_{i}^{1}(\theta)\right]$ and $\mathbf{u}_{2}=\Re\left[r^{\alpha_{j}} \mathbf{v}_{j}^{1}(\theta)\right]$

We have: $\exists C>0:\left|\nabla \mathbf{u}_{1}\right| \leq C r^{\Re \alpha_{i}-1},\left|\nabla \mathbf{u}_{2}\right| \leq C r^{\Re \alpha_{j}-1}$.

Then, $\exists C>0:\left|\Theta\left(\mathbf{u}_{1}, \mathbf{u}_{2}\right)\right| \leq C r^{\Re \alpha_{i}+\Re \alpha_{j}-2}$.

Now, with Lemma 2.1. $\Re \alpha_{i}+\Re \alpha_{j}-2>-1$. Then applying Lebesgue's Theorem, we get

$$
\int_{\partial \mathcal{H}_{D \varepsilon}} \Theta\left(\mathbf{u}_{1}, \mathbf{u}_{2}\right) d \boldsymbol{\gamma} \underset{\varepsilon \rightarrow 0}{\longrightarrow} \int_{\partial \mathcal{H}_{D}} \Theta\left(\mathbf{u}_{1}, \mathbf{u}_{2}\right) d \boldsymbol{\gamma}, \quad \int_{\partial \mathcal{H}_{N \varepsilon}} \Theta\left(\mathbf{u}_{1}, \mathbf{u}_{2}\right) d \boldsymbol{\gamma} \underset{\varepsilon \rightarrow 0}{\longrightarrow} \int_{\partial \mathcal{H}_{N}} \Theta\left(\mathbf{u}_{1}, \mathbf{u}_{2}\right) d \boldsymbol{\gamma}
$$

Moreover, there exists $C>0$ such that

$$
\left|\int_{\tilde{\Gamma}_{\varepsilon}} \Theta\left(\mathbf{u}_{1}, \mathbf{u}_{2}\right) d \gamma\right|=\varepsilon\left|\int_{0}^{\omega} \Theta\left(\mathbf{u}_{1}, \mathbf{u}_{2}\right) d \theta\right| \leq C \varepsilon^{\Re \alpha_{i}+\Re \alpha_{j}-1} \underset{\varepsilon \rightarrow 0}{\longrightarrow} 0
$$

since $\Re \alpha_{i}+\Re \alpha_{j}-1>0$. Then 13 is satisfied.

Remark. We get a similar result with $\mathbf{u}_{1}, \mathbf{u}_{2}$ or both in the form $\Re\left[r^{\alpha_{i}} \ln (r) \mathbf{v}_{i}^{2}(\theta)\right]$. 


\section{Proof of Lemma 2.1}

This Lemma has been proved in a different form especially in [13, 10].

Assume that a solution of (11) $\alpha=\gamma+i \eta$ is such that $\gamma \in(0,1 / 2)$.

We have: $\quad \sin ^{2}(\alpha \omega)=\frac{1-\cos (2 \alpha \omega)}{2}=\frac{1}{2}(1-\cos (2 \gamma \omega) \cosh (2 \eta \omega)+i \sin (2 \gamma \omega) \sinh (2 \eta \omega))$.

Moreover, $\quad \frac{4(1-\nu)^{2}-\alpha^{2} \sin ^{2} \omega}{3-4 \nu}=\frac{4(1-\nu)^{2}-\left(\gamma^{2}-\eta^{2}+2 i \gamma \eta\right) \sin ^{2} \omega}{3-4 \nu}$.

We then get

$$
\left\{\begin{aligned}
(3-4 \nu)(1-\cos (2 \gamma \omega) \cosh (2 \eta \omega)) & =2\left(4(1-\nu)^{2}+\left(\eta^{2}-\gamma^{2}\right) \sin ^{2} \omega\right) \\
(3-4 \nu) \sin (2 \gamma \omega) \sinh (2 \eta \omega) & =-4 \gamma \eta \sin ^{2} \omega
\end{aligned}\right.
$$

The first equation of (14) is even with respect to $\eta$, the second is odd. We conclude that, if $\alpha$ is a solution of 11 , then $\bar{\alpha}$ also is.

We then assume in the following $0<\gamma \leq 1 / 2$ and $\eta \geq 0$. Let us rewrite the second equation of (14)

$$
(3-4 \nu) \sin (2 \gamma \omega) \sinh (2 \eta \omega)+4 \gamma \eta \sin ^{2} \omega=0 .
$$

Let us remind that $0<\nu<1 / 2$ and $0<\omega<\pi$.

We then have in 15

$$
\left\{\begin{aligned}
(3-4 \nu) \sin (2 \gamma \omega) \sinh (2 \eta \omega) & \geq 0 \\
\gamma \eta \sin ^{2} \omega & \geq 0
\end{aligned}\right.
$$

And

$$
\left\{\begin{aligned}
(3-4 \nu) \sin (2 \gamma \omega) \sinh (2 \eta \omega) & =0 \\
\gamma \eta \sin ^{2} \omega & =0
\end{aligned}\right.
$$

Hence $\eta=0$, and the first equation of 14 gives

$$
(3-4 \nu) \cos (2 \gamma \omega)+8(1-\nu)^{2}-(3-4 \nu)-2 \gamma^{2} \sin ^{2} \omega=0 .
$$

Since $3-4 \nu>0$, for every $\gamma \in(0,1 / 2]$, we have

$$
\begin{aligned}
0 \geq(3-4 \nu) \cos \omega+8(1-\nu)^{2}-(3-4 \nu)-\frac{1}{2} \sin ^{2} \omega & =\frac{1}{2}\left(\cos ^{2} \omega+2(3-4 \nu) \cos \omega+16 \nu^{2}-24 \nu+9\right. \\
& =\frac{1}{2}(\cos \omega+(3-4 \nu))^{2}>0
\end{aligned}
$$

Which is impossible.

\subsection{Second case: $\omega=\pi$}

- Structure of the solution. Once again, we use 4, 9, 6].

In this case, equation (11) becomes

$$
\sin ^{2}(\alpha \pi)=\frac{4(1-\nu)^{2}-\alpha^{2}}{3-4 \nu}
$$

The roots of 16$)$ such that $\Re \alpha \in(0,1]$ are: $\quad \alpha=\frac{1}{2}+i k$ and $\bar{\alpha}$, with: $\quad k=\frac{\ln (3-4 \nu)}{2 \pi}$.

Then the singular solution of 10 is: $\mathbf{u}_{S}(r, \theta)=\Re\left(r^{\alpha} \mathbf{v}(\theta)\right)$. 
We need to know exactly $\mathbf{u}_{S}$. To this end, we introduce the following intermediate functions

$$
\begin{aligned}
& a(\theta)=\nu_{0}\left[\sin \left(-\frac{3}{2} \theta\right)-\sin \left(\frac{1}{2} \theta\right)-2 k\left(\cos \left(-\frac{3}{2} \theta\right)-\cos \left(\frac{1}{2} \theta\right)\right)\right] \exp (k \theta), \\
& b(\theta)=\nu_{0}\left[\cos \left(-\frac{3}{2} \theta\right)-\cos \left(\frac{1}{2} \theta\right)+2 k\left(\sin \left(-\frac{3}{2} \theta\right)-\sin \left(\frac{1}{2} \theta\right)\right)\right] \exp (k \theta), \\
& m(\theta)=4\left(\nu_{0}+2\right) \sin \left(\frac{1}{2} \theta\right) \cosh (k \theta), \\
& n(\theta)=4\left(\nu_{0}+2\right) \cos \left(\frac{1}{2} \theta\right) \sinh (k \theta),
\end{aligned}
$$

where $\nu_{0}=\frac{\lambda+\mu}{\mu}$.

We can write

$$
\mathbf{u}_{S}(r, \theta)=\sqrt{r}\left[\cos (k \ln (r))\left(\begin{array}{c}
a(\theta)-m(\theta) \\
b(\theta)+n(\theta)
\end{array}\right)+\sin (k \ln (r))\left(\begin{array}{c}
-b(\theta)+n(\theta) \\
a(\theta)+m(\theta)
\end{array}\right)\right] .
$$

Then we get: $\exists ! \tilde{\mathbf{u}}_{R} \in \mathbb{H}^{2}(\mathcal{H}), \exists ! c_{S} \in \mathbb{R}: \tilde{\mathbf{u}}=\tilde{\mathbf{u}}_{R}+c_{S} \mathbf{u}_{S}$, and then, if we write $\mathbf{u}_{R}=\mathbf{U}+\tilde{\mathbf{u}}_{R}$, we have

$$
\mathbf{u}=\mathbf{u}_{R}+c_{S} \mathbf{u}_{S}
$$

- End of the proof of Theorem 2.1 in the second case. As well as in paragraph 2.1. we use (18) and expand $\Theta(\mathbf{u}, \mathbf{u})$. Similarly, terms where $\mathbf{u}_{R}$ appears vanish.

We now consider the remaining term, quadratic with respect to $\mathbf{u}_{S}$.

Since $\omega=\pi$, we have $(\mathbf{m} \cdot \boldsymbol{\nu})=0$, on $\partial \mathcal{H}$.

Hence, $\sigma\left(\mathbf{u}_{S}\right) \boldsymbol{\nu}=0$, on $\partial \mathcal{H}_{N}$. Then $\Theta\left(\mathbf{u}_{S}, \mathbf{u}_{S}\right)=0$, on $\partial \mathcal{H}_{N}$.

Moreover, $\mathbf{u}_{S}=0$, on $\partial \mathcal{H}_{D}$. This implies that $(\mathbf{m} \cdot \nabla) \mathbf{u}_{S}=(\mathbf{m} \cdot \boldsymbol{\nu})(\boldsymbol{\nu} \cdot \nabla) \mathbf{u}_{S}$.

Then $\Theta\left(\mathbf{u}_{S}, \mathbf{u}_{S}\right)=(\mathbf{m} \cdot \boldsymbol{\nu})\left(2\left(\sigma\left(\mathbf{u}_{S}\right) \boldsymbol{\nu}\right) \cdot(\boldsymbol{\nu} \cdot \nabla) \mathbf{u}_{S}-\sigma\left(\mathbf{u}_{S}\right): \epsilon\left(\mathbf{u}_{S}\right)\right)=0$ on $\partial \mathcal{H}_{D}$.

It only remains to compute the integral on $\tilde{\Gamma}_{\varepsilon}$.

For a given $\varepsilon>0$, A technical computation gives: $\int_{\partial \Gamma_{\varepsilon}} \Theta\left(\mathbf{u}_{S}, \mathbf{u}_{S}\right) d \boldsymbol{\gamma}=\Upsilon \mathbf{m}(0) \cdot \boldsymbol{\tau}(0)+O(\varepsilon)$.

We then get: $\lim _{\varepsilon \rightarrow 0} \int_{\tilde{\Gamma}_{\varepsilon}} \Theta(\mathbf{u}, \mathbf{u}) d \boldsymbol{\gamma}=\left|c_{S}\right|^{2} \lim _{\varepsilon \rightarrow 0} \int_{\tilde{\Gamma}_{\varepsilon}} \Theta\left(\mathbf{u}_{S}, \mathbf{u}_{S}\right) d \boldsymbol{\gamma}=\Upsilon\left|c_{S}\right|^{2} \mathbf{m}(0) \cdot \boldsymbol{\tau}(0)$.

This achieves the proof of Theorem 2.1

\section{The case of a smooth bi-dimensional domain}

We here study the case of a bounded connected domain $\Omega \subset \mathbb{R}^{2}$. We assume that its boundary $\partial \Omega$ is of class $\mathcal{C}^{2}$ and satisfies (1) and (6) (In (6), $\mathbf{s}_{1}$ and $\mathbf{s}_{2}$ are two points of $\partial \Omega$ (see Figure 4).).

We assume moreover that there exists $\mathbf{x}_{0} \in \mathbb{R}^{2}$ such that (7) is satisfied. At each point $\mathbf{s}_{i}$, we denote by $\boldsymbol{\tau}\left(\mathbf{s}_{i}\right)$ the unit tangent vector to $\partial \Omega$ pointing toward $\partial \Omega_{D}$.

It can be observed that

$$
\mathbf{m}\left(\mathbf{s}_{1}\right) \cdot \boldsymbol{\nu}\left(\mathbf{s}_{1}\right)=\mathbf{m}\left(\mathbf{s}_{2}\right) \cdot \boldsymbol{\nu}\left(\mathbf{s}_{2}\right)=0 .
$$

In this case, the Rellich-type relation can be written as follows:

Theorem 3.1 - Let $\Omega \subset \mathbb{R}^{2}$ be a bounded connected domain of class $\mathcal{C}^{2}$ which satisfies (1), (6) and 19. . Let $\mathbf{u} \in \mathbb{H}^{1}(\Omega)$ be the solution of problem (2) with

$$
\mathbf{f} \in \mathbb{L}^{2}(\Omega), \quad \mathbf{g} \in \mathbb{H}^{3 / 2}\left(\partial \Omega_{D}\right), \quad \mathbf{h} \in \mathbb{H}^{1 / 2}\left(\partial \Omega_{N}\right) .
$$

Then $\Theta(\mathbf{u}, \mathbf{u})$ belongs to $\mathrm{L}^{1}(\partial \Omega)$ and

$$
2 \int_{\Omega} \operatorname{div}(\sigma(\mathbf{u})) \cdot((\mathbf{m} \cdot \nabla) \mathbf{u}) d \mathbf{x}=\int_{\partial \Omega} \Theta(\mathbf{u}, \mathbf{u}) d \boldsymbol{\gamma}+\Upsilon \sum_{\mathbf{s} \in\left\{\mathbf{s}_{1}, \mathbf{s}_{2}\right\}} c(\mathbf{s})^{2}(\mathbf{m}(\mathbf{s}) \cdot \boldsymbol{\tau}(\mathbf{s})),
$$

where $c(\mathbf{s})$ is the singularity coefficient of $\mathbf{u}$ at $\mathbf{s}$.

Once again, we need to know the structure of the studied functions. 


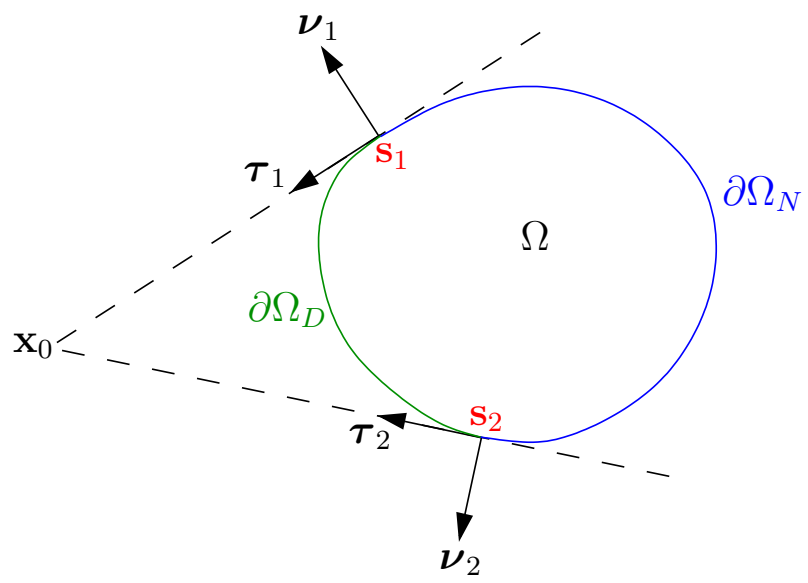

Figure 4: a smooth bi-dimensional domain $\Omega$ with a non-empty interface.

\subsection{The case of the semi-disk: structure of the solution}

We first study the case $\Omega=D^{+}(\rho)$, where $\rho>0$ so that in polar coordinates, we may define $D^{+}(\rho)=$ $(0, \rho) \times(0, \pi), \partial D_{N}^{+}(\rho)=(0, \rho) \times\{\pi\}$ and $\partial D_{D}^{+}(\rho)=(0, \rho) \times\{0\} \cup\{\rho\} \times(0, \pi)$, (see Figure 5) and we consider the problem

$$
\begin{cases}-\operatorname{div}(\sigma(\mathbf{u}))=\mathbf{f}, & \text { in } D^{+}(\rho) \\ \mathbf{u}=\mathbf{0}, & \text { on } \partial D_{D}^{+}(\rho) \\ \sigma(\mathbf{u}) \boldsymbol{\nu}=\mathbf{0}, & \text { on } \partial D_{N}^{+}(\rho)\end{cases}
$$

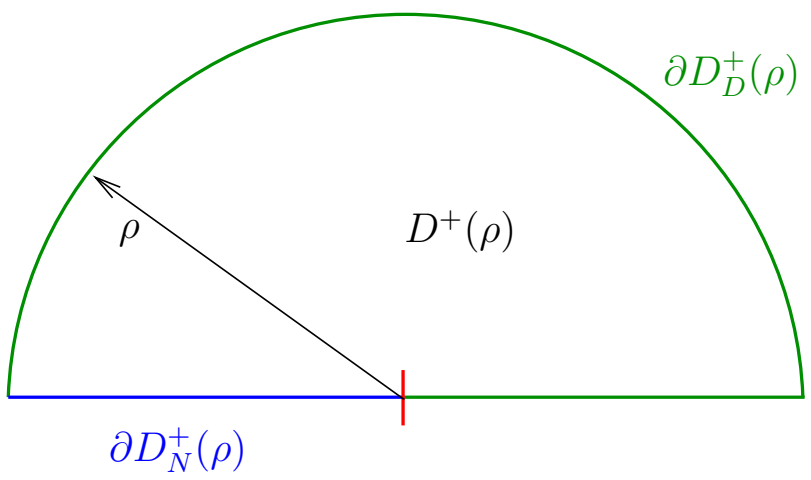

Figure 5: the considered domain is a semi-disk.

We denote by $\tilde{\mathbf{u}}_{S}$ the function defined by 17 in the whole half-plane. Let $\varsigma$ be a cut-off function belonging to $\mathcal{C}^{\infty}\left(\mathbb{R}^{+},[0,1]\right)$ such that $\operatorname{supp}(\varsigma) \subset\left[0, \rho_{2}\right]$ and $\varsigma \equiv 1$ on $\left[0, \rho_{1}\right]$, with $0<\rho_{1}<\rho_{2}<\rho$. We can define

$$
\mathbf{u}_{S}(r, \theta)=\tilde{\mathbf{u}}_{S}(r, \theta) \varsigma(r) .
$$

Using results of paragraph 2.2 we get

Proposition 3.1 - Let $\mathbf{u}$ be the solution of problem where $\mathbf{f} \in \mathbb{L}^{2}\left(D^{+}(\rho)\right)$. We have

$$
\exists ! \mathbf{u}_{R} \in \mathbb{H}^{2}\left(D^{+}(\rho)\right), \exists ! c_{S} \in \mathbb{R}: \mathbf{u}=\mathbf{u}_{R}+c_{S} \mathbf{u}_{S}
$$

where $\mathbf{u}_{S}$ is defined by 22 .

This Proposition means that, if we define the operator $\mathcal{A}_{\rho}$ by

$D\left(\mathcal{A}_{\rho}\right)=\left\{\mathbf{v} \in \mathbb{H}_{D}^{1}\left(D^{+}(\rho)\right) / \mathcal{A}_{\rho} \mathbf{v} \in \mathbb{L}^{2}\left(D^{+}(\rho)\right)\right.$ and $\sigma(\mathbf{v}) \boldsymbol{\nu}=\mathbf{0}$, on $\left.\partial D_{N}^{+}(\rho)\right\} \quad$ and $\quad \mathcal{A}_{\rho} \mathbf{v}=-\operatorname{div}(\sigma(\mathbf{v}))$, 
we get: $\quad D\left(\mathcal{A}_{\rho}\right) \subset \mathbb{H}^{2}\left(D^{+}(\rho)\right) \oplus \mathbb{R} \mathbf{u}_{S}$.

We want now to characterize the singular coefficient $c_{S}$ according to f. To this end, we introduce the following intermediate functions

$$
\begin{aligned}
& \tilde{a}(\theta)=\nu_{0}\left[\sin \left(-\frac{5}{2} \theta\right)-\sin \left(-\frac{1}{2} \theta\right)+2 k\left(\cos \left(-\frac{5}{2} \theta\right)-\cos \left(-\frac{1}{2} \theta\right)\right)\right] \exp (k \theta), \\
& \tilde{b}(\theta)=\nu_{0}\left[\cos \left(-\frac{5}{2} \theta\right)-\cos \left(-\frac{1}{2} \theta\right)-2 k\left(\sin \left(-\frac{5}{2} \theta\right)-\sin \left(-\frac{1}{2} \theta\right)\right)\right] \exp (k \theta), \\
& \tilde{m}(\theta)=4\left(\nu_{0}+2\right) \sin \left(-\frac{1}{2} \theta\right) \cosh (k \theta), \\
& \tilde{n}(\theta)=4\left(\nu_{0}+2\right) \cos \left(-\frac{1}{2} \theta\right) \sinh (k \theta),
\end{aligned}
$$

where $k$ and $\nu_{0}$ have been introduced in subsection 2.2 .

We then define

$$
\boldsymbol{\Sigma}^{*}(r, \theta)=\frac{\cos (k \ln (r))}{\sqrt{r}}\left(\begin{array}{c}
\tilde{a}(\theta)+\tilde{m}(\theta) \\
\tilde{b}(\theta)-\tilde{n}(\theta)
\end{array}\right)+\frac{\sin (k \ln (r))}{\sqrt{r}}\left(\begin{array}{c}
-\tilde{b}(\theta)-\tilde{n}(\theta) \\
\tilde{a}(\theta)-\tilde{m}(\theta)
\end{array}\right) .
$$

In the distributions sense, $\boldsymbol{\Sigma}^{*}$ is a $\mathbb{L}^{2}$ solution of the following problem

$$
\begin{cases}-\operatorname{div}(\sigma(\mathbf{u}))=\mathbf{0}, & \text { in } D^{+}(\rho) \\ \mathbf{u}=\mathbf{0}, & \text { on }(0, \rho) \times\{0\} \\ \sigma(\mathbf{u}) \boldsymbol{\nu}=\mathbf{0}, & \text { on } \partial D_{N}^{+}(\rho)\end{cases}
$$

Let us now consider $\mathbf{g}(\theta)=\boldsymbol{\Sigma}^{*}(\rho, \theta)$, for every $\theta \in(0, \pi)$. We have $\mathbf{g} \in \mathcal{C}^{\infty}([0, \pi])$. Let $\boldsymbol{\Psi}^{*} \in \mathbb{H}^{1}\left(D^{+}(\rho)\right)$ be a weak solution of the following problem

$$
\begin{cases}-\operatorname{div}(\sigma(\mathbf{u}))=\mathbf{0}, & \text { in } D^{+}(\rho) \\ \mathbf{u}=\mathbf{0}, & \text { on }(0, \rho) \times\{0\} \\ \mathbf{u}=\mathbf{g}, & \text { on }\{\rho\} \times(0, \pi) \\ \sigma(\mathbf{u}) \boldsymbol{\nu}=\mathbf{0}, & \text { on } \partial D_{N}^{+}(\rho) .\end{cases}
$$

We define $\mathbf{S}^{*}=\boldsymbol{\Sigma}^{*}-\mathbf{\Psi}^{*}$. $\mathbf{S}^{*}$ is then, in sense of distributions, a $\mathbb{L}^{2}$ solution of the following problem

$$
\begin{cases}-\operatorname{div}(\sigma(\mathbf{u}))=\mathbf{0}, & \text { in } D^{+}(\rho) \\ \mathbf{u}=\mathbf{0}, & \text { on } \partial D_{D}^{+}(\rho), \\ \sigma(\mathbf{u}) \boldsymbol{\nu}=\mathbf{0}, & \text { on } \partial D_{N}^{+}(\rho) .\end{cases}
$$

We get the following result

Proposition $3.2-$ For $\mathbf{f} \in \mathbb{L}^{2}\left(D^{+}(\rho)\right)$, let $\mathbf{u}$ be the solution of problem 21) and let $c_{S}$ be the singular coefficient of this solution. There exists $\varkappa \in \mathbb{R}^{*}$, independent of $\mathbf{f}$, such that

$$
c_{S}=\frac{1}{\varkappa} \int_{D^{+}(\rho)} \mathbf{S}^{*} \cdot \mathbf{f} d \mathbf{x} .
$$

Proof. Let $\left(\mathbf{v}_{n}\right)_{n \in \mathbb{N}} \in \mathbb{H}_{D}^{1}\left(D^{+}(\rho)\right)^{\mathbb{N}}$ be such that $\left(\mathbf{v}_{n}\right)$ tends to $\mathbf{S}^{*}$ in $\mathbb{L}^{2}\left(D^{+}(\rho)\right)$. For all $n \in \mathbb{N}$, we have

$$
\int_{D^{+}(\rho)} \sigma\left(\mathbf{u}_{R}\right): \epsilon\left(\mathbf{v}_{n}\right) d \mathbf{x}+c_{S} \int_{D^{+}(\rho)} \sigma\left(\mathbf{u}_{S}\right): \epsilon\left(\mathbf{v}_{n}\right) d \mathbf{x}=\int_{D^{+}(\rho)} \mathbf{f} \cdot \mathbf{v}_{n} d \mathbf{x} .
$$

Now, we have of course

$$
\int_{D^{+}(\rho)} \mathbf{f} \cdot \mathbf{v}_{n} d \mathbf{x} \underset{n \rightarrow \infty}{\longrightarrow} \int_{D^{+}(\rho)} \mathbf{f} \cdot \mathbf{S}^{*} d \mathbf{x} .
$$


Moreover,

$$
\int_{D^{+}(\rho)} \sigma\left(\mathbf{u}_{R}\right): \epsilon\left(\mathbf{v}_{n}\right) d \mathbf{x}=-\int_{D^{+}(\rho)} \operatorname{div}\left(\sigma\left(\mathbf{u}_{R}\right)\right) \cdot \mathbf{v}_{n} d \mathbf{x} \underset{n \rightarrow \infty}{\longrightarrow}-\int_{D^{+}(\rho)} \operatorname{div}\left(\sigma\left(\mathbf{u}_{R}\right)\right) \cdot \mathbf{S}^{*} d \mathbf{x}=0,
$$

since $\mathbf{u}_{R}=\mathbf{u}-c_{S} \mathbf{u}_{S}$ satisfies the homogeneous Neumann condition on $\partial D_{N}^{+}(\rho)$.

Then, there exists $\varkappa \in \mathbb{R}$ such that

$$
\int_{D^{+}(\rho)} \sigma\left(\mathbf{u}_{S}\right): \epsilon\left(\mathbf{v}_{n}\right) d \mathbf{x} \underset{n \rightarrow \infty}{\longrightarrow} \varkappa .
$$

We then get

$$
\varkappa c_{S}=\int_{D^{+}(\rho)} \mathbf{S}^{*} \cdot \mathbf{f} d \mathbf{x} .
$$

Now, $\varkappa$ is independent of $\mathbf{f}$. Thus $\varkappa \in \mathbb{R}^{*}$ and the Theorem is proved.

We now want to get an estimate of the $\mathbb{H}^{2}$-norm of the regular part according to $\mathbf{f}$.

Proposition 3.3 - For $\mathbf{f} \in \mathbb{L}^{2}\left(D^{+}(\rho)\right)$, let $\mathbf{u} \in D\left(\mathcal{A}_{\rho}\right)$ be the solution of problem (21). Let us denote its regular part by $\mathbf{u}_{R} \in \mathbb{H}^{2}\left(D^{+}(\rho)\right)$.

There exists $C>0$ independent of $\mathbf{f}$ such that

$$
\left\|\mathbf{u}_{R}\right\|_{\mathbb{H}^{2}\left(D^{+}(\rho)\right)} \leq C\|\mathbf{f}\|_{\mathbb{L}^{2}\left(D^{+}(\rho)\right)} .
$$

Proof. We define $\mathbb{L}_{R}^{2}\left(D^{+}(\rho)\right)=\left\{\mathbf{f} \in \mathbb{L}^{2}\left(D^{+}(\rho)\right) / \mathbf{u} \in \mathbb{H}^{2}\left(D^{+}(\rho)\right)\right\}$. Using Proposition 3.2, we may observe that the map $\mathbf{f} \mapsto c_{S}$ is linear and continuous.

Let us denote by $\mathbb{L}_{R}^{2}\left(D^{+}(\rho)\right)$ its kernel which is a closed linear space, and define

$$
\begin{aligned}
T: \mathbb{L}_{R}^{2}\left(D^{+}(\rho)\right) & \longrightarrow D\left(\mathcal{A}_{\rho}\right) \cap \mathbb{H}^{2}\left(D^{+}(\rho)\right) \\
\mathbf{f}_{R} & \longmapsto \mathbf{u}_{R} .
\end{aligned}
$$

$T$ is continuous linear and one-to-one. Moreover, we have $T^{-1}\left(\mathbf{u}_{R}\right)=-\operatorname{div}\left(\sigma\left(\mathbf{u}_{R}\right)\right)$, for every $\mathbf{u}_{R}$ in $D\left(\mathcal{A}_{\rho}\right) \cap \mathbb{H}^{2}\left(D^{+}(\rho)\right)$. We now apply the open-mapping Theorem,

$$
\exists C>0: \quad \forall \mathbf{u}_{R} \in D\left(\mathcal{A}_{\rho}\right) \cap \mathbb{H}^{2}\left(D^{+}(\rho)\right), \quad\left\|\operatorname{div}\left(\sigma\left(\mathbf{u}_{R}\right)\right)\right\|_{\mathbb{L}_{R}^{2}\left(D^{+}(\rho)\right)} \leq C\left\|\mathbf{u}_{R}\right\|_{\mathbb{H}^{1}\left(D^{+}(\rho)\right)} .
$$

Consider now

$$
\begin{aligned}
\tilde{T}: \quad D\left(\mathcal{A}_{\rho}\right) \cap \mathbb{H}^{2}\left(D^{+}(\rho)\right) & \longrightarrow \mathbb{L}_{R}^{2}\left(D^{+}(\rho)\right) \\
\mathbf{u}_{R} & \longmapsto-\operatorname{div}\left(\sigma\left(\mathbf{u}_{R}\right)\right) .
\end{aligned}
$$

$\tilde{T}$ is continuous, linear and one-to-one. Moreover, we can easily see that $D\left(\mathcal{A}_{\rho}\right) \cap \mathbb{H}^{2}\left(D^{+}(\rho)\right)$ is a Banach space with the norm induced by $\mathbb{H}^{2}\left(D^{+}(\rho)\right)$. Once again we apply the open-mapping Theorem,

$$
\exists C>0: \quad \forall \mathbf{u}_{R} \in D\left(\mathcal{A}_{\rho}\right) \cap \mathbb{H}^{2}\left(D^{+}(\rho)\right), \quad\left\|\mathbf{u}_{R}\right\|_{\mathbb{H}^{2}\left(D^{+}(\rho)\right)} \leq C\left\|\operatorname{div}\left(\sigma\left(\mathbf{u}_{R}\right)\right)\right\|_{\mathbb{L}_{R}^{2}\left(D^{+}(\rho)\right)} .
$$

Let us consider $\mathbf{f} \in \mathbb{L}^{2}\left(D^{+}(\rho)\right)$ and $\mathbf{u} \in D\left(\mathcal{A}_{\rho}\right)$, the corresponding solution of 21 ). Let $\mathbf{u}_{R} \in \mathbb{H}^{2}\left(D^{+}(\rho)\right)$ and $c_{S} \in \mathbb{R}$ be such that $\mathbf{u}=\mathbf{u}_{R}+c_{S} \mathbf{u}_{S}$. Writing the variational formulation with $\mathbf{v}=\mathbf{u}_{R}$, we get

$$
\int_{D^{+}(\rho)} \sigma\left(\mathbf{u}_{R}\right): \epsilon\left(\mathbf{u}_{R}\right) d \mathbf{x}+c_{S} \int_{D^{+}(\rho)} \sigma\left(\mathbf{u}_{S}\right): \epsilon\left(\mathbf{u}_{R}\right) d \mathbf{x}=\int_{D^{+}(\rho)} \mathbf{f} \cdot \mathbf{u}_{R} d \mathbf{x} .
$$

Using Theorem 3.2 , there exists some constant $C>0$ independent of $\mathbf{f}$ such that $\left|c_{S}\right| \leq C\|\mathbf{f}\|_{\mathbb{L}^{2}\left(D^{+}(\rho)\right)}$. Then there exists $C>0$ independent of $\mathbf{f}$ such that

$$
\left|c_{S} \int_{D^{+}(\rho)} \sigma\left(\mathbf{u}_{S}\right): \epsilon\left(\mathbf{u}_{R}\right) d \mathbf{x}\right| \leq C\|\mathbf{f}\|_{\mathbb{L}^{2}\left(D^{+}(\rho)\right)}\left\|\mathbf{u}_{R}\right\|_{\mathbb{H}^{1}\left(D^{+}(\rho)\right)} .
$$

Moreover, we get easily the existence of $c>0$ independent of $\mathbf{f}$ such that

$$
\int_{D^{+}(\rho)} \sigma\left(\mathbf{u}_{R}\right): \epsilon\left(\mathbf{u}_{R}\right) d \mathbf{x} \geq c\left\|\mathbf{u}_{R}\right\|_{\mathbb{H}^{1}\left(D^{+}(\rho)\right)}^{2} .
$$


At last, we have

$$
\left|\int_{D^{+}(\rho)} \mathbf{f} \cdot \mathbf{u}_{R} d \mathbf{x}\right| \leq\|\mathbf{f}\|_{\mathbb{L}^{2}\left(D^{+}(\rho)\right)}\left\|\mathbf{u}_{R}\right\|_{\mathbb{H}^{1}\left(D^{+}(\rho)\right)} .
$$

Then, there exists $C>0$ independent of $\mathbf{f}$ such that

$$
\left\|\mathbf{u}_{R}\right\|_{\mathbb{H}^{1}\left(D^{+}(\rho)\right)} \leq C\|\mathbf{f}\|_{\mathbb{L}^{2}\left(D^{+}(\rho)\right)} .
$$

We deduce Proposition 3.3 from (23), 24) and (25).

\subsection{Structure of the solution in the general case}

We now consider the case of a bounded connected domain $\Omega$ satisfying geometrical assumptions given in the introduction of this paragraph. As well as in the case of the polygon, the solution of problem (2) will be locally $\mathbb{H}^{2}$ in the neighborhood of every point of $\Omega$ and of every point of the boundary, except the points of $\Gamma$. We will then work at these points.

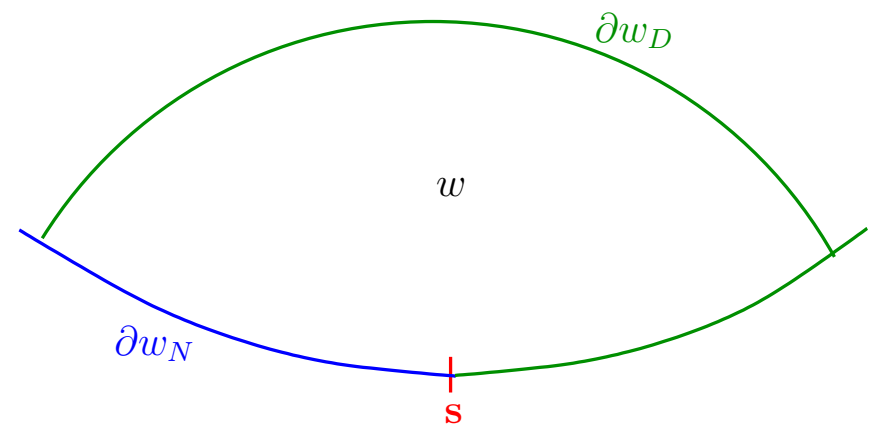

Figure 6: an example of domain induced by a neighborhood of $\mathbf{s}$.

Let then $\mathbf{s}$ be one of the two points of the interface, and let $W \subset \mathbb{R}^{2}$ be a neighborhood of $\mathbf{s}$. We denote (see Figure 6)

$$
w=W \cap \Omega, \quad \partial w_{N}=\bar{W} \cap \partial \Omega_{N}, \quad \partial w_{D}=\left(\bar{W} \cap \partial \Omega_{D}\right) \cup(\partial w \cap \Omega) .
$$

Using a localization process leads us to consider the following problem

$$
\begin{cases}-\operatorname{div}(\sigma(\mathbf{u}))=\mathbf{g}, & \text { in } w \\ \mathbf{u}=\mathbf{0}, & \text { on } \partial w_{D} \\ \sigma(\mathbf{u}) \boldsymbol{\nu}=\mathbf{0}, & \text { on } \partial w_{N}\end{cases}
$$

where $\mathbf{g} \in \mathbb{L}^{2}(w)$.

For this problem, we define the operator $\mathcal{B}$ by

$$
D(\mathcal{B})=\left\{\mathbf{v} \in \mathbb{H}_{D}^{1}(w) / \mathcal{B} \mathbf{v} \in \mathbb{L}^{2}(w) \text { and } \sigma(\mathbf{v}) \boldsymbol{\nu}=\mathbf{0} \text {, on } \partial w_{N}\right\} \text { and } \mathcal{B} \mathbf{v}=-\operatorname{div}(\sigma(\mathbf{v}))
$$

We assume that $w$ is such that there exists a $\mathcal{C}^{2}$-diffeomorphism $\phi$ from $w$ onto $D^{+}(\rho)$ for some $\rho>0$, such that

$$
\boldsymbol{\phi}(\mathbf{s})=\mathbf{0}, \quad \boldsymbol{\phi}\left(\partial w_{D}\right)=\partial D_{D}^{+}(\rho), \quad \phi\left(\partial w_{N}\right)=\partial D_{N}^{+}(\rho) .
$$

Without any restriction, we suppose that $\mathbf{s}$ is located at origin and that $x_{1}$-axis is tangent to $w$ at this point. Reducing $w$ if necessary, there exists $\psi \in \mathcal{C}^{2}(\mathbb{R}, \mathbb{R})$ such that $\partial \Omega \cap W \subset\{(x, \psi(x)) / x \in \mathbb{R}\}$. Let us consider the following function $\phi$,

$$
\phi_{1}\left(x_{1}, x_{2}\right)=x_{1}, \quad \phi_{2}\left(x_{1}, x_{2}\right)=x_{2}-\psi\left(x_{1}\right) .
$$

Using these local coordinates, 27) can be written as follows

$$
\begin{cases}-\operatorname{div}(\tilde{\sigma}(\tilde{\mathbf{u}}))=\tilde{\mathbf{g}}, & \text { in } D^{+}(\rho), \\ \tilde{\mathbf{u}}=\mathbf{0}, & \text { on } \partial D_{D}^{+}(\rho), \\ \tilde{\sigma}(\tilde{\mathbf{u}}) \boldsymbol{\nu}=\mathbf{0}, & \text { on } \partial D_{N}^{+}(\rho),\end{cases}
$$


where $\tilde{\mathbf{g}} \in \mathbb{L}^{2}\left(D^{+}(\rho)\right)$ and where $\tilde{\sigma}=\sigma+\tilde{\beta}$ with

$$
\begin{aligned}
& \tilde{\beta}_{11}(\tilde{\mathbf{u}})=-(2 \mu+\lambda) \psi^{\prime}\left(x_{1}\right) \partial_{2} \tilde{u}_{1}, \\
& \tilde{\beta}_{12}(\tilde{\mathbf{u}})=-\mu \psi^{\prime}\left(x_{1}\right) \partial_{2} \tilde{u}_{2}, \\
& \tilde{\beta}_{21}(\tilde{\mathbf{u}})=(2 \mu+\lambda) \psi^{\prime}\left(x_{1}\right)^{2} \partial_{2} \tilde{u}_{1}-(2 \mu+\lambda) \psi^{\prime}\left(x_{1}\right) \partial_{1} \tilde{u}_{1}-(\mu+\lambda) \psi^{\prime}\left(x_{1}\right) \partial_{2} \tilde{u}_{2}, \\
& \tilde{\beta}_{22}(\tilde{\mathbf{u}})=\mu \psi^{\prime}\left(x_{1}\right)^{2} \partial_{2} \tilde{u}_{2}-\mu \psi^{\prime}\left(x_{1}\right) \partial_{1} \tilde{u}_{2}-(\mu+\lambda) \psi^{\prime}\left(x_{1}\right) \partial_{2} \tilde{u}_{1} .
\end{aligned}
$$

As well as in [1, we consider problem $(29)$ as a perturbation of problem $(21)$ in order to get the following regularity result. This result generalizes Proposition 3.1

Proposition 3.4 - Let $w$ be an open subset of $\mathbb{R}^{2}$ defined in $(26)$, there exist $\rho>0$ and a $\mathcal{C}^{2}$ diffeomorphism $\phi$ from $w$ onto $D^{+}(\rho)$ such that 28 is satisfied and

$$
D(\mathcal{B}) \subset \mathbb{H}^{2}(w) \oplus \mathbb{R}\left(\mathbf{u}_{S} \circ \phi\right),
$$

where $\mathbf{u}_{S}$ is given by 22 .

Proof. We take $\rho$ and $\phi$ as above.

Let $\tilde{\mathcal{A}}_{\rho}$ be the operator defined by

$D\left(\tilde{\mathcal{A}}_{\rho}\right)=\left\{\tilde{\mathbf{v}} \in \mathbb{H}_{D}^{1}\left(D^{+}(\rho)\right) / \tilde{\mathcal{A}}_{\rho} \tilde{\mathbf{v}} \in \mathbb{L}^{2}\left(D^{+}(\rho)\right)\right.$ and $\tilde{\sigma}(\tilde{\mathbf{v}}) \boldsymbol{\nu}=0$, on $\left.\partial D_{N}^{+}(\rho)\right\} \quad$ and $\quad \tilde{\mathcal{A}}_{\rho} \tilde{\mathbf{v}}=-\operatorname{div}(\tilde{\sigma}(\tilde{\mathbf{v}}))$

We need the following Lemma, which gives estimates of the perturbation.

Lemma 3.1 Let $\rho_{m}$ be a fixed real positive number. For $\rho \in\left(0, \rho_{m}\right)$ and $\mathbf{v} \in D\left(\mathcal{A}_{\rho}\right)$, $\tilde{\beta}(\mathbf{v})$ belongs to $\mathrm{H}^{1}\left(D^{+}(\rho)\right)^{4}$ and there exists $C>0$, independent of $\rho$, such that

$$
\forall \mathbf{v} \in D\left(\mathcal{A}_{\rho}\right), \quad\|\tilde{\beta}(\mathbf{v})\|_{\mathrm{H}^{1}\left(D^{+}(\rho)\right)^{4}} \leq C \sqrt{\rho}\|\mathbf{v}\|_{D\left(\mathcal{A}_{\rho}\right)} .
$$

This Lemma is proved at the end of this section. Let us now use it.

Consider a solution $\tilde{\mathbf{u}}$ of $(29)$ belonging to $D\left(\mathcal{A}_{\rho}\right)$. Using lemma 3.1 , we get that $\tilde{\beta}(\tilde{\mathbf{u}})$ belongs to $\mathrm{H}^{1}\left(D^{+}(\rho)\right)^{4}$. Using a classical trace result, we can build $\tilde{\mathbf{u}}^{r} \in \mathbb{H}^{2}\left(D^{+}(\rho)\right)$ such that

$$
\tilde{\mathbf{u}}^{r}=\mathbf{0} \text { on } \partial D_{D}^{+}(\rho), \quad \sigma\left(\tilde{\mathbf{u}}^{r}\right) \boldsymbol{\nu}=-\tilde{\beta}(\tilde{\mathbf{u}}), \text { on } \partial D_{N}^{+}(\rho)
$$

and such that there exists $C>0$, independent of $\rho$ and $\tilde{\mathbf{u}}$, so that

$$
\left\|\tilde{\mathbf{u}}^{r}\right\|_{\mathbb{H}^{2}\left(D^{+}(\rho)\right)} \leq C\|\tilde{\beta}(\tilde{\mathbf{u}})\|_{\mathrm{H}^{1}\left(D^{+}(\rho)\right)^{4}}
$$

We can write problem $(29)$ as follows,

$$
\begin{cases}-\operatorname{div}(\sigma(\tilde{\mathbf{u}}))-\operatorname{div}(\tilde{\beta}(\tilde{\mathbf{u}}))-\operatorname{div}\left(\sigma\left(\tilde{\mathbf{u}}^{r}\right)\right)=\tilde{\mathbf{g}}, & \text { in } D^{+}(\rho), \\ \tilde{\mathbf{u}}=\mathbf{0}, & \text { on } \partial D_{D}^{+}(\rho), \\ \sigma(\tilde{\mathbf{u}}) \boldsymbol{\nu}=\mathbf{0}, & \text { on } \partial D_{N}^{+}(\rho) .\end{cases}
$$

We now define the operator $\mathcal{P}_{\rho}$ by

$$
\begin{aligned}
\mathcal{P}_{\rho}: D\left(\mathcal{A}_{\rho}\right) & \longrightarrow \mathbb{L}^{2}\left(D^{+}(\rho)\right) \\
\tilde{\mathbf{v}} & \longmapsto-\operatorname{div}(\tilde{\beta}(\tilde{\mathbf{v}}))-\operatorname{div}\left(\sigma\left(\tilde{\mathbf{v}}^{r}\right)\right) .
\end{aligned}
$$

Using Lemma 3.1. we get that $\mathcal{P}_{\rho}$ is continuous and that its norm is bounded by $C \sqrt{\rho}$, where $C$ is independent of $\rho$. Furthermore, $\mathcal{A}_{\rho}$ is an isomorphism from $D\left(\mathcal{A}_{\rho}\right)$ onto $\mathbb{L}^{2}\left(D^{+}(\rho)\right)$. Hence, for $\rho>0$ small enough, $\tilde{\mathcal{A}}_{\rho}=\mathcal{A}_{\rho}+\mathcal{P}_{\rho}$ is an isomorphism from $D\left(\mathcal{A}_{\rho}\right)$ onto $\mathbb{L}^{2}\left(D^{+}(\rho)\right)$.

Proposition 3.4 is then proved. 


\section{Proof of lemma 3.1}

For $\rho \in\left(0, \rho_{m}\right)$ and $\mathbf{v} \in D\left(\mathcal{A}_{\rho}\right)$, let us prove that $\tilde{\beta}_{11}(\mathbf{v})$ belongs to $\mathrm{H}^{1}\left(D^{+}(\rho)\right)$.

Since $\mathbf{v} \in D\left(\mathcal{A}_{\rho}\right)$, there exists $c_{S} \in \mathbb{R}$ and $\mathbf{v}_{R} \in \mathbb{H}^{2}\left(D^{+}(\rho)\right)$ such that $\mathbf{v}=\mathbf{v}_{R}+c_{S} \mathbf{u}_{S}$.

For $i \in\{1,2\}$, we have

$$
\partial_{i} \tilde{\beta}_{11}(\mathbf{v})=\partial_{i} \tilde{\beta}_{11}\left(\mathbf{v}_{R}\right)+c_{S} \partial_{i} \tilde{\beta}_{11}\left(\mathbf{u}_{S}\right) .
$$

- The term depending on $\mathbf{v}_{R}$ satisfies

$$
\partial_{i} \tilde{\beta}_{11}\left(\mathbf{v}_{R}\right)=-(2 \mu+\lambda) \partial_{i} \psi^{\prime}\left(x_{1}\right) \partial_{2} \mathbf{v}_{R 1}-(2 \mu+\lambda) \psi^{\prime}\left(x_{1}\right) \partial_{i 2} \mathbf{v}_{R 1} .
$$

Since $\psi^{\prime}(0)=0$ and $\rho \in\left(0, \rho_{m}\right)$, there exists $M>0$ independent of $\rho$ such that $\left|\psi^{\prime}\left(x_{1}\right)\right| \leq M \rho$. Then there exists $C>0$ independent of $\rho$ such that

$$
\left\|(2 \mu+\lambda) \psi^{\prime}\left(x_{1}\right) \partial_{i 2} \mathbf{v}_{R 1}\right\|_{\mathrm{L}^{2}} \leq C \rho\left\|\mathbf{v}_{R}\right\|_{\mathbb{H}^{2}} .
$$

Thanks to Proposition 3.3 , there exists $C>0$ independent of $\rho$ such that

$$
\left\|(2 \mu+\lambda) \psi^{\prime}\left(x_{1}\right) \partial_{i 2} \mathbf{v}_{R 1}\right\|_{L^{2}} \leq C \rho\|\mathbf{v}\|_{D\left(\mathcal{A}_{\rho}\right)} .
$$

On the other hand, since $\mathbf{v}$ and $\mathbf{u}_{S}$ satisfy boundary conditions, $\mathbf{v}_{R}$ satisfy them. Then $\partial_{1} \mathbf{v}_{R}$ satisfy a Dirichlet condition on $\partial D_{D}^{+}(\rho)$. Thanks to Proposition 3.3 there exists $C>0$ independent of $\rho$ such that

$$
\left\|\partial_{1} \mathbf{v}_{R}\right\|_{\mathbb{L}^{2}} \leq C \rho\|\mathbf{v}\|_{D\left(\mathcal{A}_{\rho}\right)},
$$

since Poincaré's constant is proportional to $\rho$. Moreover, $\sigma_{12}\left(\mathbf{v}_{R}\right)$ and $\sigma_{22}\left(\mathbf{v}_{R}\right)$ satisfy a Dirichlet condition on $\partial D_{N}^{+}(\rho)$. Finally we get $C>0$ independent of $\rho$ such that

$$
\left\|\partial_{2} \mathbf{v}_{R}\right\|_{\mathbb{L}^{2}} \leq C \rho\|\mathbf{v}\|_{D\left(\mathcal{A}_{\rho}\right)},
$$

and there exists $C>0$ independent of $\rho$ such that

$$
\left\|(2 \mu+\lambda) \partial_{i} \psi^{\prime}\left(x_{1}\right) \partial_{2} \mathbf{v}_{R 1}\right\|_{\mathrm{L}^{2}} \leq C \rho\|\mathbf{v}\|_{D\left(\mathcal{A}_{\rho}\right)} .
$$

Hence we can write $\left\|\partial_{i} \tilde{\beta}_{11}\left(\mathbf{v}_{R}\right)\right\|_{\mathrm{L}^{2}}=O\left(\rho\|\mathbf{v}\|_{D\left(\mathcal{A}_{\rho}\right)}\right)$.

- Let us now consider the remaining term depending on $\mathbf{u}_{S}$. Again, we have

$$
\partial_{i} \tilde{\beta}_{11}\left(c_{S} \mathbf{u}_{S}\right)=-(2 \mu+\lambda) c_{S} \partial_{i} \psi^{\prime}\left(x_{1}\right) \partial_{2} \mathbf{u}_{S 1}-(2 \mu+\lambda) c_{S} \psi^{\prime}\left(x_{1}\right) \partial_{i 2} \mathbf{u}_{S 1} .
$$

For this terms, we know $\mathbf{u}_{S}$ explicitly. We then get $C>0$ independent of $\rho$ such that

$$
\left\|(2 \mu+\lambda) c_{S} \partial_{i} \psi^{\prime}\left(x_{1}\right) \partial_{2} \mathbf{u}_{S 1}\right\|_{\mathrm{L}^{2}} \leq C \sqrt{\rho}\left|c_{S}\right|
$$

and there exists $C>0$ independent of $\rho$ such that

$$
\left\|(2 \mu+\lambda) c_{S} \psi^{\prime}\left(x_{1}\right) \partial_{i 2} \mathbf{u}_{S 1}\right\|_{\mathrm{L}^{2}} \leq C \sqrt{\rho}\left|c_{S}\right| .
$$

Now, using Proposition 3.2 we obtain $C>0$ independent of $\rho$ such that $\left|c_{S}\right| \leq C\|\mathbf{v}\|_{D\left(\mathcal{A}_{\rho}\right)}$.

And we can write $\left\|\partial_{i} \tilde{\beta}_{11}\left(c_{S} \mathbf{u}_{S}\right)\right\|_{\mathrm{L}^{2}}=O\left(\sqrt{\rho}\|\mathbf{v}\|_{D\left(\mathcal{A}_{\rho}\right)}\right)$.

Finally, we get: $\tilde{\beta}_{11}(\mathbf{v})$ belongs to $\mathrm{H}^{1}\left(D^{+}(\rho)\right)$, and there exists $C>0$ independent of $\rho$ such that

$$
\left\|\tilde{\beta}_{11}(\mathbf{v})\right\|_{\mathrm{H}^{1}\left(D^{+}(\rho)\right)} \leq C \sqrt{\rho}\|\mathbf{v}\|_{D\left(\mathcal{A}_{\rho}\right)} .
$$

We proceed similarly for other coefficients $\tilde{\beta}_{i j}(\mathbf{v})$ and we obtain the required result. 


\subsection{Proof of the Rellich-type relation}

We can now prove Theorem 3.1 .

Let $\mathbf{u} \in \mathbb{H}^{1}(\Omega)$ be the solution of problem $(2)$ with data satisfying $(20)$.

Let us proceed as well as in the polygonal case. We first use trace Theorem and build $\tilde{\mathbf{u}} \in \mathbb{H}^{2}(\Omega)$ such that $\tilde{\mathbf{u}}=\mathbf{u}$ and $\sigma(\tilde{\mathbf{u}}) \boldsymbol{\nu}=\sigma(\mathbf{u}) \boldsymbol{\nu}$ on $\partial \Omega$.

Then $\mathbf{U}=\tilde{\mathbf{u}}-\mathbf{u}$ satisfies

$$
\begin{cases}-\operatorname{div}(\sigma(\mathbf{U}))=\mathbf{F}, & \text { in } \Omega \\ \mathbf{U}=\mathbf{0}, & \text { on } \partial \Omega_{D} \\ \sigma(\mathbf{U}) \boldsymbol{\nu}=\mathbf{0}, & \text { on } \partial \Omega_{N}\end{cases}
$$

where $\mathbf{F}=\operatorname{div}(\sigma(\tilde{\mathbf{u}}))-\operatorname{div}(\sigma(\mathbf{u})) \in \mathbb{L}^{2}(\Omega)$.

We take $\varepsilon>0$ small enough and we define

$$
\begin{array}{ll}
\Omega_{\varepsilon}=\Omega \backslash\left(D\left(\mathbf{s}_{1}, \varepsilon\right) \cup D\left(\mathbf{s}_{2}, \varepsilon\right)\right), & \tilde{\Gamma}_{\varepsilon}=\left(\partial D\left(\mathbf{s}_{1}, \varepsilon\right) \cup \partial D\left(\mathbf{s}_{2}, \varepsilon\right)\right) \cap \Omega, \\
\partial \Omega_{D \varepsilon}=\partial \Omega_{D \varepsilon} \backslash\left(D\left(\mathbf{s}_{1}, \varepsilon\right) \cup D\left(\mathbf{s}_{2}, \varepsilon\right)\right), & \partial \Omega_{N \varepsilon}=\partial \Omega_{N \varepsilon} \backslash\left(D\left(\mathbf{s}_{1}, \varepsilon\right) \cup D\left(\mathbf{s}_{2}, \varepsilon\right)\right),
\end{array}
$$

so that we have $\partial \Omega_{\varepsilon}=\partial \Omega_{D \varepsilon} \cup \partial \Omega_{N \varepsilon} \cup \tilde{\Gamma}_{\varepsilon}$.

For $\mathbf{s} \in \Gamma$, we apply Proposition 3.4 to $\mathbf{U}$ in $D(\mathbf{s}, \varepsilon) \cap \Omega$. We obtain $\mathbf{U}_{R} \in \mathbb{H}^{2}(D(\mathbf{s}, \varepsilon) \cap \Omega)$ and $c_{S} \in \mathbb{R}$ such that $\mathbf{U}=\mathbf{U}_{R}+c_{S}\left(\mathbf{u}_{S} \circ \phi\right)$. Writing $\mathbf{u}_{R}=\mathbf{U}_{R}+\tilde{\mathbf{u}}$, we get

$$
\mathbf{u}=\mathbf{u}_{R}+c_{S}\left(\mathbf{u}_{S} \circ \boldsymbol{\phi}\right), \quad \text { in } D(\mathbf{s}, \varepsilon) \cap \Omega, \quad \text { with } \mathbf{u}_{R} \in \mathbb{H}^{2}(D(\mathbf{s}, \varepsilon) \cap \Omega) \text {. }
$$

Using this form, we expand $\Theta(\mathbf{u}, \mathbf{u})$.

Terms containing $\mathbf{u}_{R}$ are obviously integrable on $\partial \Omega \cap D(\mathbf{s}, \varepsilon)$.

Let us consider terms depending only on $\mathbf{u}_{S}$.

Observe that on $\partial \Omega \cap D(\mathbf{s}, \varepsilon),|(\mathbf{m} \cdot \boldsymbol{\nu})| \leq C d(., \mathbf{s})$.

By a computation, we get that $\left|(\mathbf{m} \cdot \boldsymbol{\nu}) \sigma\left(\mathbf{u}_{S} \circ \boldsymbol{\phi}\right): \epsilon\left(\mathbf{u}_{S} \circ \boldsymbol{\phi}\right)\right|$ is bounded on $\partial \Omega \cap D(\mathbf{s}, \varepsilon)$.

Now, $\left(\sigma\left(\mathbf{u}_{S} \circ \boldsymbol{\phi}\right) \boldsymbol{\nu}\right) \cdot\left((\mathbf{m} \cdot \nabla) \mathbf{u}_{S} \circ \boldsymbol{\phi}\right)$ is of course integrable on $\partial \Omega_{N} \cap D(\mathbf{s}, \varepsilon)$, since it vanishes.

On $\partial \Omega_{D} \cap D(\mathbf{s}, \varepsilon)$, since $\mathbf{u}_{S} \circ \phi=0$, we have $(\mathbf{m} \cdot \nabla) \mathbf{u}_{S} \circ \phi=(\mathbf{m} \cdot \boldsymbol{\nu})(\boldsymbol{\nu} \cdot \nabla) \mathbf{u}_{S} \circ \phi$.

Hence, $\left|\left(\sigma\left(\mathbf{u}_{S} \circ \boldsymbol{\phi}\right) \boldsymbol{\nu}\right) \cdot(\mathbf{m} \cdot \nabla) \mathbf{u}_{S} \circ \boldsymbol{\phi}\right|$ is bounded on $\partial \Omega_{D} \cap D(\mathbf{s}, \varepsilon)$.

We then obtain that $\Theta(\mathbf{u}, \mathbf{u})$ belongs to $\mathbb{L}^{1}(\partial \Omega \cap D(\mathbf{s}, \varepsilon))$. Hence, it belongs to $\mathbb{L}^{1}(\partial \Omega)$.

Now, since $\mathbf{u} \in \mathbb{H}^{2}\left(\Omega_{\varepsilon}\right)$, we apply twice Green's formula and we obtain

$$
\int_{\Omega_{\varepsilon}} \operatorname{div}(\sigma(\mathbf{u})) \cdot((\mathbf{m} \cdot \nabla) \mathbf{u}) d \mathbf{x}=\int_{\partial \Omega_{\varepsilon}} \Theta(\mathbf{u}, \mathbf{u}) d \boldsymbol{\gamma}
$$

Using Lebesgue's Theorem, we get

$\int_{\Omega_{\varepsilon}} \operatorname{div}(\sigma(\mathbf{u})) \cdot((\mathbf{m} \cdot \nabla) \mathbf{u}) d \mathbf{x} \underset{\varepsilon \rightarrow 0}{\longrightarrow} \int_{\Omega} \operatorname{div}(\sigma(\mathbf{u})) \cdot((\mathbf{m} \cdot \nabla) \mathbf{u}) d \mathbf{x}, \quad \int_{\partial \Omega_{\varepsilon} \backslash \tilde{\Gamma}_{\varepsilon}} \Theta(\mathbf{u}, \mathbf{u}) d \boldsymbol{\gamma} \underset{\varepsilon \rightarrow 0}{\longrightarrow} \int_{\partial \Omega} \Theta(\mathbf{u}, \mathbf{u}) d \boldsymbol{\gamma}$

For the remaining integral on $\tilde{\Gamma}_{\varepsilon}$, we observe that $\left|J(\phi)-I_{2}\right|=O(\varepsilon)$. By a similar computation as at the end of section 2 , we get

$$
\int_{\tilde{\Gamma}_{\varepsilon}} \Theta(\mathbf{u}, \mathbf{u}) d \gamma \underset{\varepsilon \rightarrow 0}{\longrightarrow} \Upsilon\left|c_{S}\right|^{2} \mathbf{m}(0) \cdot \boldsymbol{\tau}(0)
$$

The Rellich-type relation is then proved.

\section{The case of a smooth $n$-dimensional domain}

We here study the case of a bounded connected domain $\Omega \subset \mathbb{R}^{n}$. We assume that its boundary $\partial \Omega$ is of class $\mathcal{C}^{2}$ and satisfies (1). We assume furthermore that $\Gamma$ is a $(n-2)$-dimensional submanifold of class $\mathcal{C}^{3}$ and there exists a neighborhood $\Omega^{\prime}$ of $\Gamma$ such that $\partial \Omega \cap \Omega^{\prime}$ is a $(n-1)$-submanifold of class $\mathcal{C}^{3}$, (see Figure 7).

We assume moreover that there exists $\mathbf{x}_{0} \in \mathbb{R}^{n}$ such that (7) is satisfied.

It can be observed that under these assumptions,

$$
\mathbf{m} \cdot \boldsymbol{\nu}=0, \quad \text { on } \Gamma .
$$




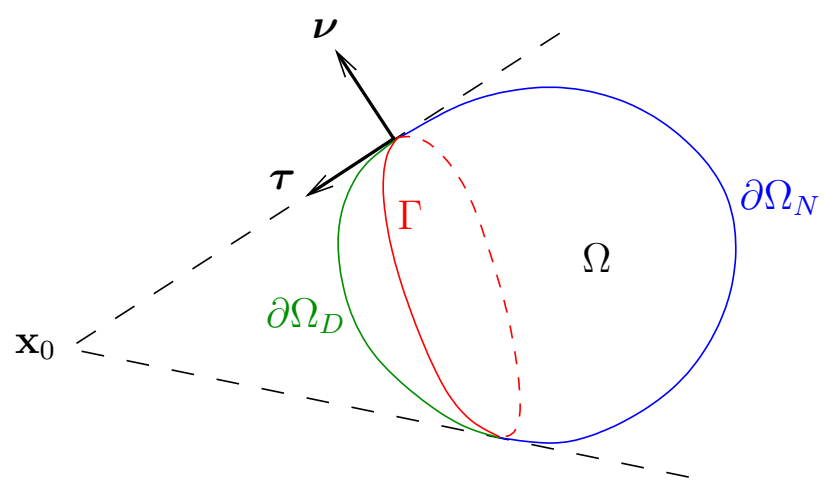

Figure 7: a general smooth domain $\Omega$ with a non-empty interface.

At each point $\mathbf{s}$ of $\Gamma$, we consider $\Gamma$ as a submanifold of $\partial \Omega$ of co-dimension 1 and we denote by $\boldsymbol{\tau}(\mathbf{s})$ the unit normal vector to $\Gamma$ pointing outward of $\partial \Omega_{N}$.

In this case, the Rellich-type relation can be written as follows.

Theorem 4.1 - Let $\Omega \subset \mathbb{R}^{n}$ be a bounded connected domain of class $\mathcal{C}^{2}$ which satisfies (1) and above geometrical assumptions. We assume moreover that the boundary satisfies $(32)$. Let $\mathbf{u} \in \mathbb{H}^{1}(\Omega)$ be the solution of problem (2) with

$$
\mathbf{f} \in \mathbb{L}^{2}(\Omega), \quad \mathbf{g} \in \mathbb{H}^{3 / 2}\left(\partial \Omega_{D}\right), \quad \mathbf{h} \in \mathbb{H}^{1 / 2}\left(\partial \Omega_{N}\right) .
$$

Then $\Theta(\mathbf{u}, \mathbf{u})$ belongs to $\mathbb{L}^{1}(\partial \Omega)$ and there exists $\zeta \in \mathrm{L}^{2}(\Gamma)$, depending on local singularity coefficients of $\mathbf{u}$, such that

$$
2 \int_{\Omega} \operatorname{div}(\sigma(\mathbf{u})) \cdot(\mathbf{m} \cdot \nabla) \mathbf{u} d \mathbf{x}=(n-2) \int_{\Omega} \sigma(\mathbf{u}): \epsilon(\mathbf{u}) d \mathbf{x}+\int_{\partial \Omega} \Theta(\mathbf{u}, \mathbf{u}) d \boldsymbol{\gamma}+\int_{\Gamma}|\zeta|^{2} \mathbf{m} \cdot \boldsymbol{\tau} d \mathbf{s} .
$$

As above, we need to know the local structure $\mathbf{u}$ at each point of $\Gamma$.

\subsection{The case of the semi-cylinder: structure of the solution}

We here use notations of section 3.1. $D^{+}(\rho)$ is the semi-disk with radius $\rho$ in dimension 2. For $d=n-2$, we here consider the case $\Omega=C^{+}(\rho)$ where $C^{+}(\rho)=D^{+}(\rho) \times \mathbb{R}^{d}$ (see Figure 8).

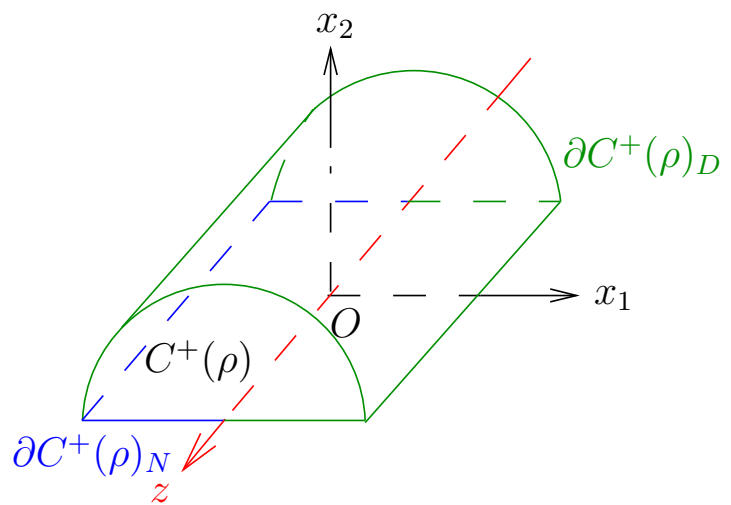

Figure 8: the considered domain is a semi-cylinder.

Coordinates of $\mathbf{x} \in C^{+}(\rho)$ will be denoted by $\left(x_{1}, x_{2}, \mathbf{z}\right)$, where $\mathbf{z}=\left(z_{1}, \ldots, z_{d}\right) \in \mathbb{R}^{d}$.

Let us define $\partial C_{N}^{+}(\rho)=\partial D_{N}^{+}(\rho) \times \mathbb{R}^{d}$ and $\partial C_{D}^{+}(\rho)=\partial D_{D}^{+}(\rho) \times \mathbb{R}^{d}$. 
We consider the problem

$$
\begin{cases}-\operatorname{div}(\sigma(\mathbf{u}))=\mathbf{f}, & \text { in } C^{+}(\rho), \\ \mathbf{u}=\mathbf{0}, & \text { on } \partial C_{D}^{+}(\rho), \\ \sigma(\mathbf{u}) \boldsymbol{\nu}=\mathbf{0}, & \text { on } \partial C_{N}^{+}(\rho) .\end{cases}
$$

Our aim is to generalize regularity results got in section 3.1

Let $\mathbf{u}_{S}^{e} \in\left(\mathrm{H}^{1}\left(D^{+}(\rho)\right)\right)^{2}$ be the singular solution defined by 22 .

Let $u_{S}^{l} \in \mathrm{H}^{1}\left(D^{+}(\rho)\right)$ be the Shamir function [12] defined in polar coordinates by

$$
u_{S}^{l}(r, \theta)=\varsigma(r) \sqrt{r} \sin \left(\frac{\theta}{2}\right)
$$

where $\varsigma$ is a cut-off function belonging to $\mathcal{C}^{\infty}\left(\mathbb{R}_{+},[0,1]\right)$ such that $\operatorname{supp}(\varsigma) \subset\left[0, \rho_{2}\right]$ and $\varsigma \equiv 1$ on $\left[0, \rho_{1}\right]$, with $0<\rho_{1}<\rho_{2}<\rho$.

Observe that $u_{S}^{l}$ is the solution of some mixed boundary problem for Laplace equation in $D^{+}(\rho)$ (see for instance [1]). It belongs to $\mathrm{H}^{1}\left(D^{+}(\rho)\right) \backslash \mathrm{H}^{2}\left(D^{+}(\rho)\right)$. We define $\mathbf{u}_{S} \in \mathbb{H}^{1}\left(D^{+}(\rho)\right)$ by

$$
\mathbf{u}_{S}={ }^{t}\left(\mathbf{u}_{S}^{e}, u_{S}^{l}, \ldots, u_{S}^{l}\right)
$$

The structure of the solution of (34) is given in the following Proposition.

Proposition 4.1 - Let $\mathbf{u}$ be the solution of problem (34). Then,

1. for every $i$ in $\{1, \ldots, d\}, \frac{\partial \mathbf{u}}{\partial z_{i}}$ belongs to $\mathbb{H}^{1}\left(C^{+}(\rho)\right)$,

2. there exists a unique element of $\mathrm{L}^{2}\left(\mathbb{R}^{d}, \mathbb{H}^{2}\left(D^{+}(\rho)\right)\right) \times \mathbb{L}^{2}\left(\mathbb{R}^{d}\right),\left(\mathbf{u}_{R}, \mathbf{c}_{S}\right)$, such that $\mathbf{u}=\mathbf{u}_{R}+\mathbf{u}_{S} \otimes \mathbf{c}_{S}$, where $\mathbf{u}_{S}$ is defined by 35 ,

3. there exists $C>0$, independent of $\rho$, such that

$$
\begin{aligned}
& \left\|\frac{\partial \mathbf{u}}{\partial z_{i}}\right\|_{\mathbb{H}^{1}\left(C^{+}(\rho)\right)} \leq C\|\mathbf{f}\|_{\mathbb{L}^{2}\left(C^{+}(\rho)\right)}, \quad \forall i \in\{1, \ldots, d\} \\
& \left\|\mathbf{u}_{R}\right\|_{\mathbb{L}^{2}\left(\mathbb{R}^{d}, \mathbb{H}^{2}\left(D^{+}(\rho)\right)\right)} \leq C\|\mathbf{f}\|_{\mathbb{L}^{2}\left(C^{+}(\rho)\right)} \\
& \left\|\mathbf{c}_{S}\right\|_{\mathbb{L}^{2}\left(\mathbb{R}^{d}\right)} \leq C\|\mathbf{f}\|_{\mathbb{L}^{2}\left(C^{+}(\rho)\right)}
\end{aligned}
$$

Proof. The first point of above Proposition and corresponding estimates can be easily obtained by using differential quotients. Hence we only develop the proof of the second one.

Notation. In order to get a more readable proof, we will say that some non-negative real number satisfies condition $C(\mathbf{f})$ if there exists $C>0$ independent of $\rho$ such that this real number is bounded by $C\|\mathbf{f}\|_{\mathbb{L}^{2}\left(C^{+}(\rho)\right)} \cdot$

Let us denote the solution of problem 34 by $\mathbf{u}={ }^{t}\left(u_{1}, \ldots, u_{n}\right)$ and define

$$
\mathbf{u}^{e}=\left(\begin{array}{l}
u_{1} \\
u_{2}
\end{array}\right) \in\left(\mathrm{H}^{1}\left(C^{+}(\rho)\right)\right)^{2} .
$$

Using $\Delta_{2}=\partial_{11}^{2}+\partial_{22}^{2}$, we rewrite 34 in the following form,

$$
\forall i \in\{3, \ldots, n\}, \quad \begin{cases}-\Delta_{2} u_{i}=g_{i}, & \text { in } C^{+}(\rho), \\ u_{i}=0, & \text { on } \partial C_{D}^{+}(\rho), \\ \partial_{2} u_{i}=h_{i}, & \text { on } \partial C_{N}^{+}(\rho)\end{cases}
$$

With the first point, we get $g_{i} \in \mathrm{L}^{2}\left(C^{+}(\rho)\right)$ and $h_{i} \in \mathrm{H}^{1 / 2}\left(C^{+}(\rho)\right)$.

Moreover $\left\|g_{i}\right\|_{\mathrm{L}^{2}\left(C^{+}(\rho)\right)}$ and $\left\|h_{i}\right\|_{\mathrm{H}^{1 / 2}\left(C^{+}(\rho)\right)}$ satisfy the condition $C(\mathbf{f})$.

Then, using [1], there exists $\left(u_{R i}, c_{S i}\right) \in \mathrm{L}^{2}\left(\mathbb{R}^{d}, \mathrm{H}^{2}\left(D^{+}(\rho)\right)\right) \times \mathrm{L}^{2}\left(\mathbb{R}^{d}\right)$ such that: $u_{i}=u_{R i}+u_{S}^{l} \otimes c_{S i}$. Furthermore, $\left\|u_{R i}\right\|_{\mathrm{L}^{2}\left(\mathbb{R}^{d}, \mathrm{H}^{2}\left(D^{+}(\rho)\right)\right)}$ and $\left\|c_{S i}\right\|_{\mathrm{L}^{2}\left(\mathbb{R}^{d}\right)}$ satisfy the condition $C(\mathbf{f})$. 
It remains to obtain the result for $\mathbf{u}^{e}$. We denote by $\operatorname{div}_{2}$ the divergence operator in $\mathbb{R}^{2}$. We also denote by $\epsilon_{2}\left(\mathbf{u}^{e}\right)$ and $\sigma_{2}\left(\mathbf{u}^{e}\right)$ the strain tensor and the stress tensor in dimension 2 . Hence, $\mathbf{u}^{e}$ satisfies

$$
\begin{cases}-\operatorname{div}_{2}\left(\sigma_{2}\left(\mathbf{u}^{e}\right)\right)=\mathbf{g}^{e}, & \text { in } C^{+}(\rho), \\ \mathbf{u}^{e}=\mathbf{0}, & \text { on } \partial C_{D}^{+}(\rho), \\ \sigma_{2}\left(\mathbf{u}^{e}\right) \boldsymbol{\nu}=\mathbf{h}^{e}, & \text { on } \partial C_{N}^{+}(\rho),\end{cases}
$$

where $\mathbf{g}^{e} \in\left(\mathrm{L}^{2}\left(C^{+}(\rho)\right)\right)^{2}, \mathbf{h}^{e} \in\left(\mathrm{H}^{1 / 2}\left(C^{+}(\rho)\right)\right)^{2}$.

Again, $\left\|\mathbf{g}^{e}\right\|_{\left(\mathrm{L}^{2}\left(C^{+}(\rho)\right)\right)^{2}}$ and $\left\|\mathbf{h}^{e}\right\|_{\left(\mathrm{H}^{1 / 2}\left(C^{+}(\rho)\right)\right)^{2}}$ satisfy the condition $C(\mathbf{f})$.

Using a trace result, we can build $\tilde{\mathbf{u}}^{e} \in\left(\mathrm{H}^{2}\left(C^{+}(\rho)\right)\right)^{2}$ such that $\left\|\tilde{\mathbf{u}}^{e}\right\|_{\left(\mathrm{H}^{2}\left(C^{+}(\rho)\right)\right)^{2}}$ satisfies the condition $C(\mathbf{f})$ and that $\mathbf{U}^{e}=\mathbf{u}^{e}-\tilde{\mathbf{u}}^{e}$ satisfies an equation in the form (37) with $\mathbf{h}^{e}=0$.

In this equation, there are no derivative with respect to $\mathbf{z}$. We then can work for a given $\mathbf{z}$, and we get that, for almost every $\mathbf{z}, \mathbf{U}^{e}$ satisfies

$$
\begin{cases}-\operatorname{div}_{2}\left(\sigma_{2}\left(\mathbf{U}^{e}(., \mathbf{z})\right)\right)=\mathbf{G}^{e}(., \mathbf{z}), & \text { in } D^{+}(\rho), \\ \mathbf{U}^{e}(., \mathbf{z})=\mathbf{0}, & \text { on } \partial D_{D}^{+}(\rho), \\ \sigma_{2}\left(\mathbf{U}^{e}(., \mathbf{z})\right) \boldsymbol{\nu}=\mathbf{0}, & \text { on } \partial C_{N}^{+}(\rho),\end{cases}
$$

where $\mathbf{G}^{e}(., \mathbf{z}) \in\left(\mathrm{L}^{2}\left(D^{+}(\rho)\right)\right)^{2}$ and there exists $C>0$ independent of $\rho$ such that

$$
\left\|\mathbf{G}^{e}(., \mathbf{z})\right\|_{\left(\mathrm{L}^{2}\left(D^{+}(\rho)\right)\right)^{2}} \leq C\|\mathbf{f}(., \mathbf{z})\|_{\left(\mathrm{L}^{2}\left(D^{+}(\rho)\right)\right)^{2}} .
$$

With Propositions 3.1, 3.2 and 3.3 we can write $\mathbf{u}^{e}(., \mathbf{z})=\mathbf{u}_{R}^{e}(., \mathbf{z})+c_{S}^{e}(\mathbf{z}) \mathbf{u}_{S}^{e}$ where $\mathbf{u}_{R}^{e}(., \mathbf{z}) \in$ $\left(\mathrm{H}^{2}\left(D^{+}(\rho)\right)\right)^{2}$. Furthermore, there exists $C>0$ independent of $\rho$ such that

$$
\left\|\mathbf{u}_{R}^{e}(., \mathbf{z})\right\|_{\left(\mathrm{H}^{2}\left(D^{+}(\rho)\right)\right)^{2}} \leq C\|\mathbf{f}(., \mathbf{z})\|_{\left(\mathrm{L}^{2}\left(D^{+}(\rho)\right)\right)^{2}},
$$

and

$$
c_{S}^{e}(\mathbf{z})=\int_{\left.D^{+}(\rho)\right)} \mathbf{S}^{*}(\mathbf{x}) \mathbf{G}^{e}(\mathbf{x}, \mathbf{z}) d \mathbf{x} .
$$

We easily get that $c_{S}^{e} \in \mathrm{L}^{2}\left(\mathbb{R}^{d}\right)$ and that $\left\|c_{S}^{e}\right\|_{\mathrm{L}^{2}\left(\mathbb{R}^{d}\right)}$ satisfies the condition $C(\mathbf{f})$.

We also get that $\mathbf{u}_{R}^{e} \in\left(\mathrm{L}^{2}\left(\mathbb{R}^{d}, \mathrm{H}^{2}\left(D^{+}(\rho)\right)\right)\right)^{2}$ and that $\left\|\mathbf{u}_{R}^{e}\right\|_{\left(\mathrm{L}^{2}\left(\mathbb{R}^{d}, \mathrm{H}^{2}\left(D^{+}(\rho)\right)\right)\right)^{2}}$ satisfies the condition $C(\mathbf{f})$. We now write

$$
\mathbf{u}_{R}={ }^{t}\left({ }^{t} \mathbf{u}_{R}^{e}, u_{R 3}, \ldots, u_{R n}\right), \quad \mathbf{c}_{S}={ }^{t}\left(c_{S}^{e}, c_{S}^{e}, c_{S 3}, \ldots, c_{S n}\right) .
$$

and we obtain the required result.

Remark. Proposition 4.1 may be expressed in other words.

For $\rho>0$, let $B_{d}(\rho)$ be the ball of $\mathbb{R}^{d}$ centered at the origin of radius $\rho$. We define

$$
\begin{array}{ll}
\tilde{C}^{+}(\rho)=D^{+}(\rho) \times B_{d}(\rho), & \tilde{\Gamma}(\rho)=\{(0,0)\} \times B_{d}(\rho), \\
\partial \tilde{C}_{N}^{+}(\rho)=\partial D_{N}^{+}(\rho) \times B_{d}(\rho), & \partial \tilde{C}_{D}^{+}(\rho)=\partial \tilde{C}^{+}(\rho) \backslash \partial \tilde{C}_{N}^{+}(\rho),
\end{array}
$$

and consider the following problem

$$
\begin{cases}-\operatorname{div}(\sigma(\mathbf{u}))=\mathbf{f}, & \text { in } \tilde{C}^{+}(\rho), \\ \mathbf{u}=\mathbf{0}, & \text { on } \partial \tilde{C}_{D}^{+}(\rho), \\ \sigma(\mathbf{u}) \boldsymbol{\nu}=\mathbf{0}, & \text { on } \partial \tilde{C}_{N}^{+}(\rho) .\end{cases}
$$

Using a cut-off function, Proposition 4.1 allows us to prove that, if we define the operator $\mathcal{A}_{\rho}$ by

$$
D\left(\mathcal{A}_{\rho}\right)=\left\{\mathbf{v} \in \mathbb{H}_{D}^{1}\left(\tilde{C}^{+}(\rho)\right) / \mathcal{A}_{\rho} \mathbf{v} \in \mathbb{L}^{2}\left(\tilde{C}^{+}(\rho)\right) \text { and } \sigma(\mathbf{v}) \boldsymbol{\nu}=\mathbf{0}, \text { on } \partial \tilde{C}_{N}^{+}(\rho)\right\}, \quad \mathcal{A}_{\rho} \mathbf{v}=-\operatorname{div}(\sigma(\mathbf{v})),
$$

we get: $\quad D\left(\mathcal{A}_{\rho}\right) \subset \mathbb{H}^{2}\left(\tilde{C}^{+}(\rho)\right) \oplus\left(\mathbf{u}_{S} \otimes \mathbb{L}^{2}\left(B_{d}(\rho)\right)\right)$.

We now can estimate of the $\mathbb{H}^{2}$-norm of the regular part according to $\mathbf{f}$.

Proposition $4.2-$ For $\mathbf{f} \in \mathbb{L}^{2}\left(\tilde{C}^{+}(\rho)\right)$, let $\mathbf{u} \in D\left(\mathcal{A}_{\rho}\right)$ be the solution of problem 40 . Its regular part, $\mathbf{u}_{R} \in \mathbb{H}^{2}\left(\tilde{C}^{+}(\rho)\right)$, satisfies: there exists $C>0$ independent of $\mathbf{f}$ such that

$$
\left\|\mathbf{u}_{R}\right\|_{\mathbb{H}^{2}\left(\tilde{C}^{+}(\rho)\right)} \leq C\|\mathbf{f}\|_{\mathbb{L}^{2}\left(\tilde{C}^{+}(\rho)\right)} .
$$

Proof. We only follow the proof of Proposition 3.3 . 


\subsection{Structure of the solution in the general case}

We now consider the case of a bounded connected domain $\Omega$ satisfying geometrical assumptions given in the introduction of this paragraph. As well as in the 2-dimensional case, the solution of problem (2) is locally $\mathbb{H}^{2}$ in the neighborhood of every point of $\Omega$ and of every point of $\partial \Omega \backslash \Gamma$.

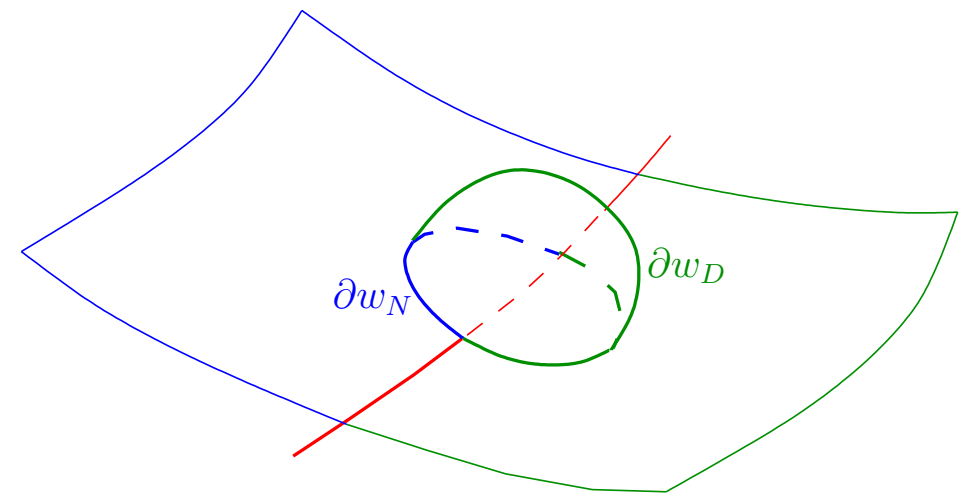

Figure 9: an example of domain induced by a neighborhood of a point of $\Gamma$.

For $\mathbf{s} \in \Gamma$, let $W \subset \mathbb{R}^{n}$ be a neighborhood of $\mathbf{s}$. We define (see Figure 9)

$$
\begin{array}{ll}
w=W \cap \Omega, & \gamma=W \cap \Gamma, \\
\partial w_{N}=W \cap \partial \Omega_{N}, & \partial w_{D}=\left(W \cap \partial \Omega_{D}\right) \cup(\partial w \cap \Omega),
\end{array}
$$

A localization process (see [1]) leads us to consider the following problem:

$$
\begin{cases}-\operatorname{div}(\sigma(\mathbf{u}))=\mathbf{g}, & \text { in } w \\ \mathbf{u}=\mathbf{0}, & \text { on } \partial w_{D}, \\ \sigma(\mathbf{u}) \boldsymbol{\nu}=\mathbf{0}, & \text { on } \partial w_{N}\end{cases}
$$

where $\mathbf{g} \in \mathbb{L}^{2}(w)$.

For this problem, we define the operator $\mathcal{B}$ by

$$
D(\mathcal{B})=\left\{\mathbf{v} \in \mathbb{H}_{D}^{1}(w) / \mathcal{B} \mathbf{v} \in \mathbb{L}^{2}(w) \text { and } \sigma(\mathbf{v}) \boldsymbol{\nu}=\mathbf{0}, \text { on } \partial w_{N}\right\}, \quad \mathcal{B} \mathbf{v}=-\operatorname{div}(\sigma(\mathbf{v})) .
$$

We assume that $w$ is such that for any $\rho>0$, there exists a $\mathcal{C}^{2}$-diffeomorphism $\boldsymbol{\Phi}$ from $w$ onto $\tilde{C}^{+}(\rho)$ which satisfies

$$
\boldsymbol{\Phi}(\mathbf{s})=\mathbf{0}, \quad \boldsymbol{\Phi}\left(\partial w_{D}\right)=\partial \tilde{C}_{D}^{+}(\rho), \quad \boldsymbol{\Phi}\left(\partial w_{N}\right)=\partial \tilde{C}_{N}^{+}(\rho), \quad \boldsymbol{\Phi}(\gamma)=\tilde{\Gamma}(\rho) .
$$

Modifying $w$ if necessary, and for simplicity, we will consider a particular $\boldsymbol{\Phi}$. As well as in subsection 3.2 . without any restriction, we suppose that the considered point $\mathbf{s}$ of $\Gamma$ is located at the origin, that $\partial w$ is tangent to $x_{2}$-axis and $\gamma$ to the subspace $\left\{x_{1}=x_{2}=0\right\}$ at this point.

Reducing $w$ if necessary, there exists $\phi \in \mathcal{C}^{2}\left(\mathbb{R}^{n-1}, \mathbb{R}\right)$ and $\boldsymbol{\psi} \in \mathcal{C}^{2}\left(\mathbb{R}^{d}, \mathbb{R}\right)$ such that

$$
\forall \mathbf{x}=\left(x_{1}, x_{2}, \mathbf{z}\right) \in w, \quad \mathbf{\Phi}(\mathbf{x})=\left(x_{1}-\boldsymbol{\psi}(\mathbf{z}), x_{2}-\phi\left(x_{1}, \mathbf{z}\right), \mathbf{z}\right)
$$

and

$$
\nabla_{\mathbf{z}} \boldsymbol{\phi}(0, \mathbf{0})=\nabla_{\mathbf{z}} \boldsymbol{\psi}(\mathbf{0})=\mathbf{0}, \quad \frac{\partial \boldsymbol{\phi}}{\partial x_{1}}(0, \mathbf{0})=\mathbf{0} .
$$

Hence, the Jacobian matrix of $\boldsymbol{\Phi}$ is given by:

$$
D \boldsymbol{\Phi}=\left(\begin{array}{ccc}
1 & 0 & -{ }^{t} \nabla_{\mathbf{z}} \boldsymbol{\partial} \\
-\frac{\partial \phi}{\partial x_{1}} & 1 & -{ }^{t} \nabla_{\mathbf{z}} \boldsymbol{\phi} \\
\mathbf{0} & \mathbf{0} & I_{d}
\end{array}\right) .
$$


Especially, $D \boldsymbol{\Phi}(\mathbf{0})=I_{n}$.

Using these local coordinates, 42 can be written as follows

$$
\begin{cases}-\operatorname{div}(\tilde{\sigma}(\tilde{\mathbf{u}}))=\tilde{\mathbf{g}}, & \text { in } \tilde{C}^{+}(\rho), \\ \tilde{\mathbf{u}}=\mathbf{0}, & \text { on } \partial \tilde{C}_{D}^{+}(\rho), \\ \tilde{\sigma}(\tilde{\mathbf{u}}) \boldsymbol{\nu}=\mathbf{0}, & \text { on } \partial \tilde{C}_{N}^{+}(\rho),\end{cases}
$$

where $\tilde{\mathbf{g}} \in \mathbb{L}^{2}\left(\tilde{C}^{+}(\rho)\right)$ and where

$$
\tilde{\sigma}(\tilde{\mathbf{u}})=\mu \nabla \boldsymbol{\Phi}\left(\nabla \tilde{\mathbf{u}} \nabla \boldsymbol{\Phi}+{ }^{t} \nabla \boldsymbol{\Phi}^{t} \nabla \tilde{\mathbf{u}}\right)+\lambda(\nabla \boldsymbol{\Phi}: \nabla \tilde{\mathbf{u}}) \nabla \boldsymbol{\Phi} .
$$

Once again, we write $\tilde{\beta}(\tilde{\mathbf{u}})=\tilde{\sigma}(\tilde{\mathbf{u}})-\sigma(\tilde{\mathbf{u}})$ and we consider $\tilde{\beta}(\tilde{\mathbf{u}})$ as a perturbation of problem 40 in order to get the following regularity result.

Proposition 4.3 - Let $w$ be an open subset of $\mathbb{R}^{n}$ defined in form (41). There exist $\rho>0$ and a $\mathcal{C}^{2}$-diffeomorphism $\mathbf{\Phi}$ from $w$ onto $\tilde{C}^{+}(\rho)$ such that 43 is satisfied and

$$
D(\mathcal{B}) \subset \mathbb{H}^{2}(w) \oplus\left(\left(\mathbf{u}_{S} \otimes \mathbb{L}^{2}\left(B_{d}(\rho)\right)\right) \circ \mathbf{\Phi}\right)
$$

where $\mathbf{u}_{S}$ is given by 35 .

Proof. We take $\rho$ and $\boldsymbol{\Phi}$ as above.

Let $\tilde{\mathcal{A}}_{\rho}$ be the operator defined by

$$
D\left(\tilde{\mathcal{A}}_{\rho}\right)=\left\{\tilde{\mathbf{v}} \in \mathbb{H}_{D}^{1}\left(C^{+}(\rho)\right) / \tilde{\mathcal{A}}_{\rho} \tilde{\mathbf{v}} \in \mathbb{L}^{2}\left(C^{+}(\rho)\right) \text { and } \tilde{\sigma}(\tilde{\mathbf{v}}) \boldsymbol{\nu}=\mathbf{0}, \text { on } \partial C_{N}^{+}(\rho)\right\}, \quad \tilde{\mathcal{A}}_{\rho} \tilde{\mathbf{v}}=-\operatorname{div}(\tilde{\sigma}(\tilde{\mathbf{v}}))
$$

The following Lemma allows us to estimate the perturbation $\tilde{\beta}=\tilde{\sigma}-\sigma$.

Lemma 4.1 - For $\rho>0$ and $\mathbf{v} \in D\left(\mathcal{A}_{\rho}\right), \tilde{\beta}(\mathbf{v})$ belongs to $\mathrm{H}^{1}\left(\tilde{C}^{+}(\rho)\right)^{n^{2}}$ and there exists $C>0$, independent of $\rho$, such that

$$
\forall \mathbf{v} \in D\left(\mathcal{A}_{\rho}\right), \quad\|\tilde{\beta}(\mathbf{v})\|_{\mathrm{H}^{1}\left(\tilde{C}^{+}(\rho)\right)^{n^{2}}} \leq C \sqrt{\rho}\|\mathbf{v}\|_{D\left(\mathcal{A}_{\rho}\right)} .
$$

Assuming that Lemma 4.1 holds, we follow the proof of Proposition 3.4 .

\section{Proof of Lemma 4.1}

Using (47), we can write, for $(i, j) \in\{1, \ldots, n\}^{2}$ and $\mathbf{v} \in D\left(\mathcal{A}_{\rho}\right)$, above functions $\tilde{\beta}_{i j}$ in the form:

$$
\tilde{\beta}_{i j}(\mathbf{v})=\sum_{k, l=1}^{n} \kappa_{k l}^{i j} \partial_{k} v_{l}
$$

where $\forall(i, j, k, l) \in\{1, \ldots, n\}^{4}, \kappa_{k l}^{i j} \in \mathcal{C}^{1}\left(\tilde{C}^{+}(\rho)\right)$ and $\kappa_{k l}^{i j}(\mathbf{0})=0$.

For $\mathbf{v} \in D\left(\mathcal{A}_{\rho}\right)$ and $t \in\{3, \ldots, n\}$, we have

$$
\partial_{t} \tilde{\beta}_{i j}(\mathbf{v})=\sum_{k, l=1}^{n} \partial_{t} \kappa_{k l}^{i j} \partial_{k} v_{l}+\sum_{k, l=1}^{n} \kappa_{k l}^{i j} \partial_{k t} v_{l} .
$$

On one hand, we use Proposition 4.1: there exists $C>0$ independent of $\rho$ such that

$$
\left\|\partial_{k t} v_{l}\right\|_{\mathrm{L}^{2}\left(\tilde{C}^{+}(\rho)\right)} \leq C\|\mathbf{v}\|_{D\left(\mathcal{A}_{\rho}\right)} .
$$

Since $\kappa_{k l}^{i j}(\mathbf{0})=0$, there exists $C>0$ independent of $\rho$ such that

$$
\left\|\sum_{k, l=1}^{n} \kappa_{k l}^{i j} \partial_{k t} v_{l}\right\|_{L^{2}\left(\tilde{C}^{+}(\rho)\right)} \leq C \rho\|\mathbf{v}\|_{D\left(\mathcal{A}_{\rho}\right)} .
$$


On the other hand, since $\mathbf{v}$ satisfies a Dirichlet condition on $\partial \tilde{C}_{D}^{+}(\rho), \partial_{k} \mathbf{v}$ satisfies also a Dirichlet condition on a part of the boundary, for $k \in\{3, \ldots, n\}$. Then, since the Poincaré's constant is proportional to $\rho$, there exists $C>0$ independent of $\rho$ such that

$$
\left\|\partial_{k} \mathbf{v}\right\|_{\mathbb{L}^{2}\left(\tilde{C}^{+}(\rho)\right)} \leq C \rho\|\mathbf{v}\|_{D\left(\mathcal{A}_{\rho}\right)} .
$$

Moreover, since $\sigma_{12}(\mathbf{v})$ and $\sigma_{22}(\mathbf{v})$ satisfy a Dirichlet condition on $\partial \tilde{C}_{N}^{+}(\rho)$, we get that there exists $C>0$ independent of $\rho$ such that

$$
\left\|\partial_{2} \mathbf{v}\right\|_{\mathbb{L}^{2}\left(\tilde{C}^{+}(\rho)\right)} \leq C \rho\|\mathbf{v}\|_{D\left(\mathcal{A}_{\rho}\right)} .
$$

We get then $C>0$ independent of $\rho$ such that

$$
\left\|\sum_{k, l=1}^{n} \partial_{t} \kappa_{k l}^{i j} \partial_{k} v_{l}\right\|_{L^{2}\left(\tilde{C}^{+}(\rho)\right)} \leq C \rho\|\mathbf{v}\|_{D\left(\mathcal{A}_{\rho}\right)} .
$$

We then have got $C>0$ independent of $\rho$ such that

$$
\left\|\partial_{t} \tilde{\beta}_{i j}(\mathbf{v})\right\|_{L^{2}(\tilde{C}+(\rho))} \leq C \rho\|\mathbf{v}\|_{D\left(\mathcal{A}_{\rho}\right)}
$$

We now assume that $t \in\{1,2\}$. Similarly, there exists $C>0$ independent of $\rho$ such that

$$
\left\|\partial_{t}\left(\sum_{k=3}^{n} \sum_{l=1}^{n} \kappa_{k l}^{i j} \partial_{k} v_{l}\right)\right\|_{L^{2}\left(\tilde{C}^{+}(\rho)\right)} \leq C \rho\|\mathbf{v}\|_{D\left(\mathcal{A}_{\rho}\right)} .
$$

Since $\mathbf{v} \in D\left(\mathcal{A}_{\rho}\right)$, there exists $\mathbf{v}_{R} \in \mathrm{L}^{2}\left(\mathbb{R}^{d}, \mathbb{H}^{2}\left(D^{+}(\rho)\right)\right)$ and $\mathbf{c}_{S} \in \mathbb{L}^{2}\left(\mathbb{R}^{d}\right)$ such that $\mathbf{v}=\mathbf{v}_{R}+\mathbf{u}_{S} \otimes \mathbf{c}_{S}$. Once again, as above, we get $C>0$ independent of $\rho$ such that

$$
\left\|\partial_{t}\left(\sum_{k=1}^{2} \sum_{l=1}^{n} \kappa_{k l}^{i j} \partial_{k} v_{R l}\right)\right\|_{\mathcal{L}^{2}\left(\tilde{C}^{+}(\rho)\right)} \leq C \rho\|\mathbf{v}\|_{D\left(\mathcal{A}_{\rho}\right)} .
$$

For the remaining term with $\mathbf{u}_{S}$, we have

$$
\partial_{t}\left(\sum_{k=1}^{2} \sum_{l=1}^{n} \kappa_{k l}^{i j} \partial_{k}\left(\mathbf{v}_{S} \otimes \mathbf{c}_{S}\right)_{l}\right)=\sum_{k=1}^{2} \sum_{l=1}^{n} \partial_{t} \kappa_{k l}^{i j}\left(\partial_{k} \mathbf{u}_{S} \otimes \mathbf{c}_{S}\right)_{l}+\sum_{k=1}^{2} \sum_{l=1}^{n} \kappa_{k l}^{i j}\left(\partial_{k t} \mathbf{u}_{S} \otimes \mathbf{c}_{S}\right)_{l}
$$

Observe that $\mathbf{u}_{S}$ is explicitly known. A technical computation gives $C>0$ independent of $\rho$ such that

$$
\left\|\sum_{k=1}^{2} \sum_{l=1}^{n} \partial_{t} \kappa_{k l}^{i j}\left(\partial_{k} \mathbf{u}_{S} \otimes \mathbf{c}_{S}\right)_{l}\right\|_{\mathrm{L}^{2}\left(\tilde{C}^{+}(\rho)\right)} \leq C \sqrt{\rho}\left\|\mathbf{c}_{S}\right\|_{\mathbb{L}^{2}}
$$

and

$$
\left\|\sum_{k=1}^{2} \sum_{l=1}^{n} \kappa_{k l}^{i j}\left(\partial_{k t} \mathbf{u}_{S} \otimes \mathbf{c}_{S}\right)_{l}\right\|_{L^{2}\left(\tilde{C}^{+}(\rho)\right)} \leq C \sqrt{\rho}\left\|\mathbf{c}_{S}\right\|_{\mathbb{L}^{2}} .
$$

Since there also exists $C>0$ independent of $\rho$ such that

$$
\left\|\mathbf{c}_{S}\right\|_{\mathbb{L}^{2}} \leq C\|\mathbf{v}\|_{D\left(\mathcal{A}_{\rho}\right)}
$$

we get $C>0$ independent of $\rho$ such that

$$
\left\|\partial_{t}\left(\sum_{k=1}^{2} \sum_{l=1}^{n} \kappa_{k l}^{i j} \partial_{k}\left(\mathbf{v}_{S} \otimes \mathbf{c}_{S}\right)_{l}\right)\right\|_{L^{2}} \leq C \sqrt{\rho}\|\mathbf{v}\|_{D\left(\mathcal{A}_{\rho}\right)} .
$$

Finally, for every $(i, j)$ in $\{1, \ldots, n\}^{2}$, we get: $\tilde{\beta}_{i j}(\mathbf{v}) \in \mathrm{H}^{1}\left(\tilde{C}^{+}(\rho)\right)$, and there exists $C>0$ independent of $\rho$ such that

Lemma 4.1 is then proved.

$$
\left\|\tilde{\beta}_{i j}\right\|_{\mathrm{H}^{1}\left(\tilde{C}^{+}(\rho)\right)} \leq C \sqrt{\rho}\|b v\|_{D\left(\mathcal{A}_{\rho}\right)} .
$$




\subsection{Proof of the Rellich-type relation}

We now can prove Theorem 4.1 .

Let $\mathbf{u} \in \mathbb{H}^{1}(\Omega)$ be the solution of problem (2) satisfying conditions (33).

Using a trace result, we build $\tilde{\mathbf{u}} \in \mathbb{H}^{2}(\Omega)$ such that $\tilde{\mathbf{u}}=\mathbf{u}$ and $\sigma(\tilde{\mathbf{u}}) \boldsymbol{\nu}=\sigma(\mathbf{u}) \boldsymbol{\nu}$ on $\partial \Omega$. Then $\mathbf{U}=\mathbf{u}-\tilde{\mathbf{u}}$ satisfies

$$
\begin{cases}-\operatorname{div}(\sigma(\mathbf{U}))=\mathbf{F}, & \text { in } \Omega \\ \mathbf{U}=\mathbf{0}, & \text { on } \partial \Omega_{D} \\ \sigma(\mathbf{U}) \boldsymbol{\nu}=\mathbf{0}, & \text { on } \partial \Omega_{N}\end{cases}
$$

where $\mathbf{F}=\operatorname{div}(\sigma(\tilde{\mathbf{u}}))-\operatorname{div}(\sigma(\mathbf{u})) \in \mathbb{L}^{2}(\Omega)$.

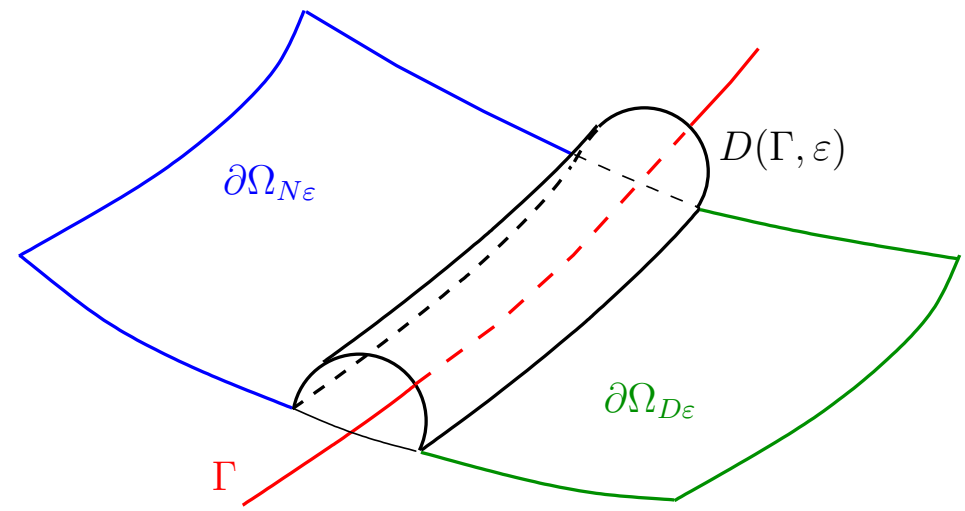

Figure 10: construction of $\Omega_{\varepsilon}$.

Let $\varepsilon>0$ be small enough and define (see Figure 10)

$$
\begin{array}{ll}
\Omega_{\varepsilon}=\Omega \backslash D(\Gamma, \varepsilon), & \tilde{\Gamma}_{\varepsilon}=\partial D(\Gamma, \varepsilon) \cap \Omega, \\
\partial \Omega_{N \varepsilon}=\partial \Omega_{N} \backslash D(\Gamma, \varepsilon), & \partial \Omega_{D \varepsilon}=\partial \Omega_{D} \backslash D(\Gamma, \varepsilon),
\end{array}
$$

where $D(\Gamma, \varepsilon)=\left\{\mathbf{x} \in \mathbb{R}^{n} / d(\mathbf{x}, \Gamma) \leq \varepsilon\right\}$. We have $\partial \Omega_{\varepsilon}=\partial \Omega_{D \varepsilon} \cup \partial \Omega_{N \varepsilon} \cup \tilde{\Gamma}_{\varepsilon}$.

As well as for Theorem 3.1 , $\Theta(\mathbf{u}, \mathbf{u})$ belongs to $\mathbb{L}^{1}(\partial \Omega)$.

Observe that $\mathbf{u} \in \mathbb{H}^{2}\left(\Omega_{\varepsilon}\right)$. We apply twice Green's formula and obtain

$$
\int_{\Omega_{\varepsilon}} \operatorname{div}(\sigma(\mathbf{u})) \cdot((\mathbf{m} \cdot \nabla) \mathbf{u}) d \mathbf{x}=(n-2) \int_{\Omega_{\varepsilon}} \sigma(\mathbf{u}): \epsilon(\mathbf{u}) d \mathbf{x}+\int_{\partial \Omega_{\varepsilon}} \Theta(\mathbf{u}, \mathbf{u}) d \boldsymbol{\gamma} .
$$

Using Lebesgue's Theorem, we get

$$
\begin{gathered}
\int_{\Omega_{\varepsilon}} \operatorname{div}(\sigma(\mathbf{u})) \cdot((\mathbf{m} \cdot \nabla) \mathbf{u}) d \mathbf{x} \underset{\varepsilon \rightarrow 0}{\longrightarrow} \int_{\Omega} \operatorname{div}(\sigma(\mathbf{u})) \cdot((\mathbf{m} \cdot \nabla) \mathbf{u}) d \mathbf{x} \\
\int_{\Omega_{\varepsilon}} \sigma(\mathbf{u}): \epsilon(\mathbf{u}) d \mathbf{x} \underset{\varepsilon \rightarrow 0}{\longrightarrow} \int_{\Omega} \sigma(\mathbf{u}): \epsilon(\mathbf{u}) d \mathbf{x} \\
\int_{\partial \Omega_{D \varepsilon} \cup \partial \Omega_{N \varepsilon}} \Theta(\mathbf{u}, \mathbf{u}) d \boldsymbol{\gamma} \underset{\varepsilon \rightarrow 0}{\longrightarrow} \int_{\partial \Omega} \Theta(\mathbf{u}, \mathbf{u}) d \boldsymbol{\gamma}
\end{gathered}
$$

It remains to study the convergence of the integral on $\tilde{\Gamma}_{\varepsilon}$. We consider $\mathbf{s} \in \Gamma$. After convenient rotation and translation, we assume that this point $\mathbf{s}$ is the origin, and that $\boldsymbol{\tau}(\mathbf{s})$ and $\boldsymbol{\nu}(\mathbf{s})$ give the first and the second axises of coordinates. We write $\tilde{\Gamma}_{\varepsilon}(\mathbf{s})=\tilde{\Gamma}_{\varepsilon} \cap(\mathbf{s}, \boldsymbol{\tau}, \boldsymbol{\nu})$ where $(\mathbf{s}, \boldsymbol{\tau}, \boldsymbol{\nu})$ is the plane containing $\mathbf{s}$ generated by $\boldsymbol{\tau}$ and $\nu$.

Applying Proposition 4.3 we get $\mathbf{u}=\mathbf{u}_{R}+\left(\mathbf{u}_{S} \circ \mathbf{\Phi}\right) \otimes \mathbf{c}_{S}(\mathbf{s})$ on some neighborhood $w(\mathbf{s})$ of $\mathbf{s}$. We have 
$\boldsymbol{\Phi}\left(\tilde{\Gamma}_{\varepsilon}\right)=\{\varepsilon\} \times(0, \pi)$ in polar coordinates.

We obtain

$$
\begin{aligned}
\int_{\tilde{\Gamma}_{\varepsilon}(\mathbf{s})} \Theta(\mathbf{u}, \mathbf{u}) d \boldsymbol{\gamma}= & \sum_{i=1}^{d}\left|c_{S i}(\mathbf{s})\right|^{2} \int_{\theta=0}^{\pi} \mu\left[\left(\boldsymbol{\nu} \cdot \nabla u_{S}^{l}\right) \cdot\left(\mathbf{m} \cdot \nabla u_{S}^{l}\right)-(\mathbf{m} \cdot \boldsymbol{\nu})\left|\nabla u_{S}^{l}\right|^{2}\right] \varepsilon d \theta \\
& +\left|c_{S}^{e}(\mathbf{s})\right|^{2} \int_{\theta=0}^{\pi} \Theta\left(\mathbf{u}_{S}^{e}, \mathbf{u}_{S}^{e}\right) \varepsilon d \theta+O(\varepsilon) .
\end{aligned}
$$

Using results of [5, 1], we get

$$
\int_{\theta=0}^{\pi}\left[\left(\boldsymbol{\nu} \cdot \nabla u_{S}^{l}\right) \cdot\left(\mathbf{m} \cdot \nabla u_{S}^{l}\right)-(\mathbf{m} \cdot \boldsymbol{\nu})\left|\nabla u_{S}^{l}\right|^{2}\right] \varepsilon d \theta=\frac{\pi}{4}(\mathbf{m}(\mathbf{s}) \cdot \boldsymbol{\tau}(\mathbf{s}))+O(\varepsilon) .
$$

Moreover, we have obtained in section 3.3 .

$$
\int_{\theta=0}^{\pi} \Theta\left(\mathbf{u}_{S}^{e}, \mathbf{u}_{S}^{e}\right) \varepsilon d \theta=\Upsilon(\mathbf{m}(\mathbf{s}) \cdot \boldsymbol{\tau}(\mathbf{s}))+O(\varepsilon) .
$$

Hence, we obtain

$$
\int_{\tilde{\Gamma}_{\varepsilon}(\mathbf{s})} \Theta(\mathbf{u}, \mathbf{u}) d \boldsymbol{\gamma}=\left[\Upsilon\left|c_{S}^{e}(\mathbf{s})\right|^{2}+\mu \frac{\pi}{4} \sum_{i=1}^{d}\left|c_{S i}(\mathbf{s})\right|^{2}\right] \mathbf{m}(\mathbf{s}) \cdot \boldsymbol{\tau}(\mathbf{s})+O(\varepsilon) .
$$

We integrate the previous result on $w(\mathbf{s}) \cap \Gamma$. Since $\Gamma$ is compact, we can introduce a finite covering of $\Gamma$ by $\{w(\mathbf{s})\}_{\mathbf{s} \in \Gamma}$ and the associated cut-off functions which constitute a partition of the unity of $\Gamma$. We then combine a finite number of equalities and we finally get the function $\zeta$ announced in Theorem 4.1.The Rellich-type relation is obtained by passing to the limit when $\varepsilon$ tends to 0 .

\section{References}

[1] Bey, R., Lohéac, J.-P., Moussaoui, M., 1999, Singularities of the solution of a mixed problem for a general second order elliptic equation and boundary stabilization of the wave equation. J. Math. pures et appli., 78, 1043-1067.

[2] Brossard, R., LohÉac, J.-P., 2004, Stabilisation frontière du système élastodynamique dans un polygone plan. C.R. Acad. Sci. Paris, série I Math., 338, 213-218.

[3] Brossard, R., LohÉac, J.-P., 2007, Boundary stabilization of elastodynamic systems. Part II: The case of a linear feedback. preprint

[4] Grisvard, P., 1986, Boundary value problems in plan polygons. Instruction for use. E.D.F., Bulletin de la Direction des Études et Recherche, série C, Mathématiques no 1, 21-59.

[5] Grisvard, P., 1989, Contrôlabilité exacte des solutions de l'équation des ondes en présence de singularités. J. Math. pures et appli., 68, 215-259.

[6] Grisvard, P., 1989, Singularités en élasticité. Arch. Rational Mech. Anal., 107, no 2, 157-180.

[7] Komornik, V., ZuazuA, E., 1990, A direct method for the boundary stabilization of the wave equation. J. Math. pures et appl., 69, 33-54.

[8] Lions, J.-L., Magenes, E., 1968, Problèmes aux limites non homogènes et leurs applications. Dunod, Paris.

[9] Mérouani, B., 1996, Solutions singulières du système de l'élasticité dans un polygone pour différentes conditions aux limites. Maghreb Math Rev., 5, no 1-2, 95-112. 
[10] NiCAise, S., 1992, About the Lamé system in a polygonal or a polyhedral domain and a coupled problem between the Lamé system and the plate equation I: Regularity of the solutions. Annali della Scuola Normale Sup. di Pisa, Série IV, 19, 327-361.

[11] Rellich, F., 1940, Darstellung der Eigenwerte von $\nabla u+\lambda u=0$ durch ein Randintegral. Math. Zeitschrift, 46, 635-636.

[12] Shamir, E., 1968, Regularity of mixed second order elliptic problems. Israel J. Math., 6, 150-168.

[13] Teniou, D., 1987, Divers problèmes théoriques et numériques liés au système de l'élasticité dans des domaines non réguliers. Thèse de doctorat. 